Revista de Psicología Vol. 36 (1), 2018 (ISSN 0254-9247)

\title{
Superando la 'duda crónica': Historia de la Psicología y formación de psicólogos argentinos en el contexto de la psicología latinoamericana ${ }^{1}$
}

\author{
Catriel Fierro ${ }^{2}$ \\ Universidad Nacional de Mar de Plata, \\ Consejo Nacional de Investigaciones Cientificas y Técnicas (CONICET) \\ Argentina
}

El presente estudio constituye un análisis empírico de la relevancia de la educación histórica de los psicólogos en el marco de la psicología latinoamericana. Se caracteriza la 'duda crónica' sobre el sentido formativo de la historiografía, describiéndose las formas en que dicha historiografía representa un insumo central en la formación de los psicólogos latinoamericanos y argentinos. Luego se exponen los resultados de un análisis cuantitativo y cualitativo descriptivo, de tipo socio-bibliométrico, sobre la literatura utilizada como bibliografía $(n=798)$ en asignaturas históricas de carreras de psicología en Argentina. Los resultados indican un predominio marcado de literatura de autores argentinos, una escasez marcada de autores latinoamericanos y una representación en ocasiones problemática del pluralismo histórico de la disciplina. Se concluye sobre las implicaciones de tales resultados para una historia

1 Una versión resumida de la presente investigación obtuvo la Mención Honorífica del Premio de Investigación 'Ignacio Martín-Baró', categoría 'Trabajo de Pregrado' en su 35ta edición, otorgada por la Sociedad Interamericana de Psicología, y fue comunicada en el contexto del XXXV Congreso Interamericano de Psicología, realizado en la Pontificia Universidad Católica de Perú, ciudad de Lima, Perú, en el mes de Julio del año 2015. El autor desea agradecer a Hugo Klappenbach, Ana María Talak, Walter Arias, Laureano Brisuela, Agostina Vorano y a un revisor anónimo por los comentarios en torno a una versión previa del trabajo. Adicionalmente, el autor desea agradecer a Modesto Alonso y a Humberto Castilla por su gentil facilitación de datos y bibliografía que contribuyeron al análisis aquí presentado. Finalmente, el autor expresa su agradecimiento a Tomás Caycho por su gentil invitación a una estadía de investigación que facilitó la socialización de este trabajo. Las conclusiones de esta investigación son sin embargo exclusiva responsabilidad del autor.

2 Licenciado en Psicología por la Universidad Nacional de Mar del Plata. Profesor por concurso de oposición y antecedentes de la asignatura Historia Social de la Psicologia. Dirección postal: Viamonte 2035 8F, Mar Del Plata, Buenos Aires, Argentina. Contacto: catriel.fierro@gmail. com 
crítica en la formación de psicólogos latinoamericanos y sobre la necesidad de contextualizar la historia local en la historia regional de la ciencia.

Palabras clave: historia de la psicología, enseñanza de la psicología, formación en psicología, pensamiento crítico, psicología latinoamericana

Overcoming the 'Chronic Doubt': History of Psychology and Argentinian Psychologists' Training and Education in the Context of Latin-American Psychology

The present study presents an empirical analysis of the relevance of psychologists' historical education within the framework of Latin American psychology. The 'chronic doubt' about the formative aim of historiography is first characterized. The ways in which such historiography is a central input in Latin American and Argentinian psychologists' training and education is then described. The results of a descriptive, mixed socio-bibliometric analysis of the literature used as readings $(n=798)$ in undergraduate historical courses at Argentinian psychology programs are presented. Findings indicate a marked predominance of texts and scholarship by Argentinian authors, a marked scarcity of Latin American authors and themes, and a problematic depiction of psychology's historical pluralism. We conclude on the implications of such results for a critical history in the Latin American psychologists' education, and on the need to contextualize local history in regional history of science.

Keywords: history of psychology, teaching of psychology, education in psychology, critical thinking, Latin-American psychology

Superando a "dúvida crônica": História da Psicologia e Formaçáo em psicologia em Argentina no contexto da psicologia latino-americana

O presente estudo constitui uma análise empírica da relevância da educação histórica dos psicólogos no quadro da psicologia latino-americana. É caracterizada a "dúvida crônica" sobre o sentido formativo da historiografia, descrevendo as formas pelas quais essa historiografia representa um aporte central no treinamento de psicólogos latino-americanos e argentinos. São apresentados os resultados de uma análise quantitativa e qualitativa descritiva, sócio-bibliométrica da literatura utilizada como bibliografia $(n=798)$ em disciplinas históricas de carreiras de psicologia na Argentina. Os resultados indicam uma marcada predominância da literatura dos autores argentinos, uma escassez marcada de autores latino-americanos e uma representação problemática do pluralismo histórico da disciplina. Conclui sobre as implicaçôes de tais resultados para uma história crítica na formação de psicólogos latino-americanos e sobre a necessidade de contextualizar a história local na história regional da ciência.

Palavras-chave: história da psicologia, ensino de psicologia, treinamento em psicologia, pensamento critico, Psicologia latino-americana.

\section{Surmonter le "doute chronique": histoire de la psychologie et formation des psycholo- gues argentins dans le contexte de la psychologie latino-américaine.}

La présente étude constitue une analyse empirique de la pertinence de l'éducation historique des psychologues dans le contexte de la psychologie latino-américaine. Le "doute chronique" sur le sens formatif de l'historiographie est caractérisé, en décrivant les manières dont cette historiographie représente un apport central dans la formation des psychologues latinoaméricains et argentins. Les résultats d'une analyse descriptive quantitative et qualitative, de type socio-bibliométrique, sur la littérature utilisée comme bibliographie $(n=798)$ dans le contenu des cours historiques de carrières en psychologie en Argentine sont présentés. 
Les résultats indiquent une prédominance marquée de la littérature écrite par des auteurs argentins, une pénurie marquée d'auteurs latino-américains et une représentation parfois problématique du pluralisme historique de la discipline. Il conclut sur les implications de tels résultats pour une histoire critique dans la formation des psychologues latino-américains et sur la nécessité de contextualiser l'histoire locale dans l'histoire régionale de la science. Mots-clé: histoire de la psychologie, enseignement de la psychologie, formation en psychologie, la pensée critique, psychologie latino-américaine.

Formación, Historia y Enseñanza de la Psicología constituyen, especialmente desde la década de 1980, tres áreas íntimamente relacionadas, a escala internacional (Buchanan, 2002) y específicamente en el ámbito de la psicología latinoamericana (Gallegos, 2016). Entre otras cosas, el campo de la formación en psicología indaga la dimensión curricular, infraestructural e institucional de los procesos de entrenamiento académico y profesional de psicólogos en el marco de carreras de grado y posgrado, tomando como objeto de estudio, por caso, los procesos de acreditación (Di Doménico, 2015; Klappenbach, 2015) las competencias y habilidades científico-profesionales específicas desarrolladas por los psicólogos-estudiantes (Gonzalez et al., 2012; Manzo, 2010; Parra Herrera, Colunga Santos \& Carvajal Hernández, 2016), las incumbencias disciplinares (Klappenbach, 2000a), y las peculiaridades y configuraciones de los perfiles de graduado de países o regiones específicas (Di Doménico, 1996; 1999; García, 2009; González, 2015a; Toro \& Villegas, 2001).

La enseńanza de la psicología como especialidad refiere tanto a la propia enseñanza-aprendizaje de la psicología en el ámbito universitario (Coll, 1996; Landrum, 2016), como a la reflexión sistemática e investigación en torno a la capacitación de profesionales en innovación y diseño curricular (Klappenbach, 2003a; Vilanova, 1996), y finalmente a la determinación de las vías concretas a partir de las cuales realimentar la profesión mejorando la formación a través de la instrucción (Fierro \& Di Doménico, 2017; Polanco \& Calabresi, 2011). Finalmente, la historia de la psicología — como objeto de estudio- 
refiere al acervo dinámico de acontecimientos, procesos, narrativas y desarrollos (teóricos, profesionales, e institucionales) referidos al pasado de la psicología, sea como sea que se entienda a esta última de acuerdo a sus diversos proyectos fundacionales (Vezzetti, 1998). A través de las operaciones investigativas del historiador sobre las evidencias que reposan en las fuentes históricas que desembocan en una narración retrospectiva y esencialmente reconstructiva, la historia como objeto de estudio, tal como es recuperada en el presente, confiere identidad y planeamiento futuro a la psicología contemporánea (Furumoto, 1989; Gimenez, 2002; Klappenbach, 2000b; Richards, 1987; Sokal, 1984b), sin esto excluir una disposición crítica hacia dicha identidad (Woodward, 1980).

En términos de Giménez (2002), la historia, la historia de la ciencia y la historia de la psicología constituyen formas "de memoria colectiva, [de] inscripción y la transmisión de las señas de pertenencia, rasgos, relaciones internas y vínculos externos, que caracterizan e identifican a las entidades grupales" (p. 215). Como actividad académica y con pretensiones de cientificidad, el campo de Historia de la Psicología está sujeta a requisitos específicos, de índole técnica y metodológica (rigor crítico, selección de fuentes, pertinencia, interpretación) y requisitos generales, de índole filosófica (conciencia del investigador respecto de sus objetivos, harmonía entre las interpretaciones históricas y el conocimiento previamente disponible, concreción lingüística de tales interpretaciones en teorías y narraciones fundamentadas, plausibles y probables). Finalmente, como campo de estudio, desde 1970 la historiografía de la psicología se ha transformado de forma constante, producto entre otras cosas de la profesionalización e institucionalización del ámbito y de la incorporación de múltiples influencias teóricas y metodológicas novedosas provenientes de campos disciplinares adyacentes (Capshew, 2014; Fierro 2016b).

Previo a que se instalase la discusión sistemática de problemas teóricos y epistemológicos en el campo de la historia de la psicología (Woodward, 1980), se inició hacia 1960 en el ámbito académico norteamericano una línea de relevamientos y análisis (conceptuales y 
empíricos) acerca de la historia de la psicología en la formación universitaria de los psicólogos; línea que se sistematizó hacia 1980 y 1990 (Hilgard, Leary \& McGuire, 1991; Ware \& Benjamin, 1991) y que llega hasta la actualidad (Beins, 2016; Benjamin, 2010; Merced, Stutman \& Mann, 2017; Rutherford \& Pickren, 2015; Vaughn-Blount, Rutherford, Baker, \& Johnson, 2009). Estos análisis se han fundamentado, en última instancia, en los argumentos de los psicólogos-historiadores acerca de la función que cumpliría la enseñanza de contenidos, cuestiones y problemáticas históricas e historiográficas en la formación académica y profesional de los psicólogos. Este es, efectivamente, uno de los puntos en que formación, enseñanza e historia convergen. Así, desde 1960, los psicólogos-historiadores comenzaron tanto a relevar los contenidos, fuentes y enfoques históricos de los cursos de grado sobre historia de la psicología, como a justificar y legitimar a través de variadas argumentaciones la medida y forma concreta en que la historia de la psicología, a partir de su inclusión curricular, podía redundar en beneficios formativos para el graduado (Collins \& Bunn, 2016).

De acuerdo a relevamientos de conjunto sobre la literatura en la temática (Fierro, 2016a; Fierro, Ostrovsky \& Di Doménico, 2017; en prensa) la reflexión conceptual y la indagación empírica de la enseñanza de la historia de la psicología en países latinoamericanos es escasa en términos comparativos, respecto tanto a la producción investigativa de la región en otras áreas no-históricas, como a la producción sobre la temática en países norteamericanos y europeos. Esto representa un problema dado que dos de los desafíos centrales que enfrentan los historiadores y pedagogos responsables de asignaturas históricas en el curriculum del psicólogo es el difundir una percepción favorable sobre la utilidad y el sentido crucial de tales asignaturas en la formación del psicólogo (Barnes \& Greer, 2016; Rutherford, 2002) junto con el facilitar una síntesis de excelencia entre la instrucción universitaria, formación científico-crítica y los avances de la subdisciplina. A la vez, esto representa un desafío dado que, en coagencia con otros factores como la reducción presupuestaria y la revisión de los curricula, el fracaso parcial de esta empresa ha llevado al estancamiento y en ocasiones al retroceso 
del ofrecimiento del curso en países norteamericanos (Bhatt $\&$ Tonks 2002; Fuchs \& Viney, 2002; Norcross, Hailstorks, Aiken \& Pfund, 2016) y europeos (Brock, 2016b; Brock \& Harvey, 2015; ChisvertPerales, Monteagudo-Soto \& Mestre, 2016; Mestre, 2007; Richards, 2005; Samper et al., 2008).

A partir de los escasos trabajos disponibles sobre la cuestión, la situación del curso de historia de la psicología en Latinoamérica que es heterogénea y varía en función de estado de la subdisciplina, de los especialistas disponibles y de las peculiaridades de los sistemas educativos de los países respectivos. De acuerdo a tales trabajos, existen razones para suponer que dicha situación es mayoritariamente problemática: en Paraguay, por caso, se ha eliminado la asignatura de Historia de la Psicología de la malla curricular de ciertas facultades (García, 2011a; 2011b). En la Universidad Nacional Autónoma de México, actualmente una de las más pobladas del continente, el curso de Historia de la Psicología fue adicionado a la curricula de psicología hacia fines de la década del año 2000 (Monroy-Nasr et al., 2009). Varias universidades peruanas incluyen cursos de historia de la psicología, aunque pocas de las mismas son enseñadas por especialistas o investigadores en cuestiones históricas (Arias \& Oblitas, 2014; Arias, en prensa), siendo Perú un país con una sostenida producción historiográfica (Arias, 2014). Finalmente, si bien en Argentina los contenidos históricos constituyen por reglamentación oficial contenidos curriculares básicos en la formación de grado del psicólogo (Fierro \& Di Doménico, 2016), futuras revisiones de conjunto sobre los estándares de los procesos de acreditación factiblemente repararán en las razones (existentes o no) para continuar considerando tales contenidos como mínimos y fundamentales.

La presente investigación se enmarca en la intersección de las tres áreas definidas en el sentido recién esbozado, en el contexto formativo específico de la Psicología latinoamericana (más precisamente, el de la Psicología del Cono Sur) y a la luz del problema que representan dos de los rasgos más sobresalientes de dicho contexto: el de la existencia de marcados sesgos y déficits de formación y entrenamiento, y el de la ausencia de análisis empíricos acerca de la historia de la psicología 
en la formación universitaria de los psicólogos argentinos. A la luz de estos rasgos, el objetivo de este trabajo es analizar críticamente la 'duda crónica' acerca del valor curricular de la historia de la psicología, vinculando significativamente el sentido de la educación histórica en ciencia, por un lado, con ciertas carencias formativas del psicólogo argentino y latinoamericano extensivamente documentadas, por otro. A partir de constatar la relación excluyente entre tales carencias y la enseñanza de la historia de la psicología, se exponen los resultados de un relevamiento empírico sobre ciertos aspectos específicos de los contenidos de la enseñanza de la historia de la psicología en universidades nacionales argentinas, especialmente en lo tocante a la presencia de autores, temáticas y corrientes de la psicología latinoamericana en asignaturas históricas, con la finalidad de apoyar las reflexiones y recomendaciones sobre la inserción curricular de la historiografía.

\section{La 'duda crónica' sobre el valor de la historia de la psicología y sus respuestas: ¿Por qué la historia importa?}

Según el historiador de la ciencia Roger Smith, el valor de la tarea que realiza el psicólogo profesional al indagar la historia de su ciencia, o el sentido de las investigaciones de historiadores profesionales no psicólogos, son a menudo objeto de cuestionamiento y suspicacia por parte de los psicólogos no-historiadores (Smith, 2007). Esta es, concretamente, la duda acerca del valor de la historia de la psicología. Smith explica esta situación aduciendo que los recursos y el tiempo disponible para las investigaciones es limitado en un sentido material e institucional, y que de acuerdo al ordenamiento de las prioridades investigativas en países como Estados Unidos y Canadá, la historia es así vista como un lujo, una pieza de cultura general o un pasatiempo, más no un legítimo ámbito de investigación científica.

Junto con este factor infraestructural, se reconoce desde hace décadas (Krantz, 1972) que por cuestiones de entrenamiento profesional y por inclinaciones hacia la investigación fáctica que en pocos casos se combina con intereses filosóficos, los psicólogos - o al menos el grueso de ellos - tienden a asumir sin cuestionar los implícitos 
de su propia ciencia. Contrariamente, el historiador de la psicología (especialmente aquel sin formación en la disciplina) a menudo realiza precisamente tal tipo de cuestionamientos, implícita o explícitamente a través de sus investigaciones, lo cual instituye una relación de tensión entre él y sus colegas (Vezzetti, 2007). Finalmente, Smith (2007) aduce que quien investiga el pasado de la psicología suele ser, en la mayoría de los casos, el propio psicólogo: no el historiador de las ideas y mucho menos el historiador profesional de la ciencia. Aunque esta imagen parece estar invirtiéndose por el avance de historiadores no psicólogos sobre el campo, especialmente en países como Canadá, Estados Unidos e Inglaterra (Barnes \& Greer, 2016; Brock, 2016a), se ha reconocido de forma consensuada la existencia de cierto 'hiato' entre psicólogos con intereses históricos, por un lado, e historiadores entrenados, por otro (Danziger, 1994, 1997; Dehue, 1998; Weidman, 2016), lo cual introduce problemas y tensiones en cuanto a los objetivos de la investigación histórica, en cuanto al canon técnico-metodológico y en cuanto a la posibilidad que los productos investigativos de la historiografía sean de utilidad o de significancia para la disciplina (Vera Ferrándiz, 2008). Por la escasez de recursos por los que compite, por su perspectiva crítica, y por apartar (supuestamente) su atención de los auténticamente legítimos problemas empíricos de la psicología, el psicólogo historiador es visto así con suspicacia. En estas condiciones "la duda acerca del valor de la historia es crónica. Se vuelve aguda, adicionalmente, cuando hay competitividad intensa, o cuando existe una reducción del apoyo financiero" (Smith, 2007, p. 129).

Tal duda crónica no se circunscribe a la psicología, sino que es ciudadana tanto en campos científicos extra-psicológicos — siendo claros ejemplos la física, la biología y la sociología - como en el propio campo disciplinar de la historia e historiografía de la ciencia (Brush, 1974; Gooday, Lynch, Wilson \& Barsky, 2008), filtrándose incluso al cuestionamiento de la relevancia de la historia y otras disciplinas de 'segundo orden', como la filosofía de la ciencia, para la formación y entrenamiento científicos (Matthews, 2015). De hecho, el cuestionamiento sobre la utilidad o sentido de la historia de la psicología en la 
socialización académica han repercutido efectivamente en las indagaciones sobre la historia de la psicología y su enseńanza universitaria (Fierro, 2015a; 2015b). Lo que es relevante aquí es la pervivencia en el tiempo de la discusión en torno al auténtico valor del estudio de la historia de la ciencia para la formación de científicos, especialmente a partir de la década de 1970 y al interior de la historia, la filosofía e incluso la sociología de la ciencia (Christie, 2005). Dado que precisamente a partir de 1970 comenzó a profesionalizarse e institucionalizarse la historia de la psicología, nutriéndose de los campos investigativos recién referidos, se sigue que junto con tales influencias, se recibió y reformuló el cuestionamiento básico latente en la 'duda crónica' acerca del valor de la historia de la ciencia y de su enseñanza para los profesionales y académicos (psicólogos) contemporáneos.

Así, los historiadores de la psicología parecen compartir con otros historiadores de la ciencia la tarea de defender y argumentar acerca del sentido de su cometido paralelamente a que realizan sus propias investigaciones: especialmente en las últimas décadas, ante la proliferación de cursos históricos en los curricula norteamericanos, y como resultado de los cuestionamientos dirigidos hacia su labor, "los historiadores de la psicología, dirigiéndose a psicólogos, han avanzado ciertos argumentos acerca de que el conocimiento sobre la ciencia del pasado colabora o apoya a la ciencia contemporánea" (Smith, 2007, p. 129). Una reciente revisión (Smith, 2016) de hecho identificaba diez argumentos sobre la valorización de la historia: en tanto práctica disciplinar, la historia serviría como una forma de celebrar, memorizar y mantener una identidad colectiva, como una via para registrar el progreso científico y el avance de valores humanos, como un medio para mantener la unidad en la diversidad en una disciplina heterogénea como la psicología, como un recurso para la práctica e investigación contemporánea, como un insumo para una perspectiva crítica de la disciplina, como una forma de contribuir al bienestar y autoconocimiento humano, como una forma sistemática de reconocer e indagar el fenómeno de la naturaleza histórica del objeto de la psicología, como una via empírica para abordar los enunciados psicológicos en el contexto del discurso 
de la disciplina (discurso que es un producto parcialmente histórico), $y$, finalmente, como un fin en sí mismo. Aunque los argumentos que explicitan la contribución de la investigación y la enseñanza de la historia de la psicología a la formación de psicólogos son numerosos y heterogéneos, y un análisis de la totalidad de tales argumentaciones excede este trabajo, tres de ellos (íntimamente vinculados entre sí) son especialmente relevantes de cara a los objetivos de este trabajo, y en su conjunto fundamentan el 'rol vital' (Goodwin, 1997) o la 'centralidad' (Rosa, 2002) de la historia en la formación de psicólogos.

Desde que se pronunciara inicialmente la necesidad de una historia crítica de la psicología (Danziger, 1984; Harris, 1980; Samelson, 1980), historiadores y pedagogos de la psicología han remarcado que la enseñanza del pasado disciplinar estimularía el pensamiento crítico en los alumnos (Bartos \& Banks, 2015; Woodward, 1980; 2010). Tal tipo de pensamiento implica, por un lado y en un sentido general independientemente del objeto de análisis crítico, la fundamentación sistemática, racional, informada y reflexiva de la propia cognición y acción (Ennis, 1991), y constituye una de las competencias básicas que persigue toda formación universitaria (Fierro \& Di Doménico, 2017; Hawes, 2003). Por otro lado, el pensamiento crítico respecto de la disciplina en su totalidad que vehiculiza la historiografía implica el asumir la contingencia del desarrollo científico a través de re-imaginar el presente, puesto que la historia "interroga y desenmaraña lo que previamente se daba por sentado al exponer su estatus de verdad cultural e históricamente contingente" (Rutherford, 2014, p. 868).

El pensamiento crítico requiere tanto de un fundamento sólido como de un cúmulo de conocimientos previos que sirvan de trasfondo e implica, entre otras cosas, el cuestionamiento de supuestos propios y ajenos, la consideración de la totalidad de fuentes informacionales disponibles, y el reconocimiento y evitación de sesgos informacionales e interpretativos. Tales lineamientos son, a nivel histórico e historiográfico, insignes de la historia crítica sistematizada a partir de 1970 (Danziger, 1984; Harris; 1980; 2011; Samelson, 1980; Woodward, 1980), y su desarrollo en alumnos se ha estimado posible a través de la enseñanza 
de contenidos históricos mediante recursos instruccionales específicos (Benjamin, 2010; Henderson, 1995; Robinson, 1979; Zehr, 2000a; 2004). La historia de la psicología, a través del análisis de fuentes primarias (Woody, 2011), a través del debate áulico (Carroll, 2006) y a través de la comparación metateórica (Carroll, Keniston \& Peden, 2008; Kushner, 1980) contribuiría con desarrollar tal tipo de pensamiento.

Junto con estimular el pensamiento crítico, la enseñanza de la historia de la psicología en la formación de grado es valiosa en tanto permite la contextualización de las teorías, corrientes y constructos del pasado de la psicología en el ambiente intelectual y en las condiciones sociales, políticas y económicas de cada época histórica (Rutherford \&Pickren, 2015; van Strien, 1990; Vilanova, 1997a), especialmente en el contexto de planes de estudio que tienden a presentar los contenidos formativos de la disciplina sin un trasfondo histórico o como elementos universales y a-históricos (Danziger, 1994). A nivel más general, tal instrucción histórica da lugar a la contextualización significativa de la psicología contemporánea en el marco general de su desarrollo: es decir, en el marco de su propio pasado (Benjamin \& Baker, 2009; Tortosa, Mayor \& Carpintero, 1990). Respecto a la contextualización socio-cultural, puesto que "la historia es acerca del contexto" (Benjamin, 2010, p. 178. Énfasis propio), una enseñanza integral de la historia de esta ciencia (y una formación básica y profesional integral) no puede prescindir de enmarcar a los autores y a las teorías de la psicología en las matrices históricas, culturales y sociales de las que necesariamente son producto: tal contextualización constituye un recurso explicativo básico tanto de la emergencia histórica de la psicología (Danziger, 2013; Young, 1966) como de la emergencia histórica de la ciencia en general (Christie, 2005). Formativamente, esta contextualización "ayuda a los estudiantes a entender cómo las temáticas psicológicas afectan y son afectadas por un mundo crecientemente interconectado" (Benjamin, 2010, p. 178).

Puesto que este argumento implica una concepción sociocultural de la actividad científica, la contextualización social a menudo es realizada a partir de enfoques de historia social o sociológica de la ciencia: es decir, enfoques con una teoría general de la acción social, 
incluso de aquellas inherentes a la ciencia (Danziger, 1984; Sokal, 1984a; Vilanova, 1997a). Tal contextualización no es inherentemente incompatible con enfoques de historia conceptual, intelectual o 'de las ideas' (van Strien, 1990), siempre que la raigambre sociocultural de los constructos y teorías no sea omitida o desertada a favor de análisis puramente intelectualistas o discursivos, dado que aplicados a la historia de la ciencia estos a menudo incurren en distorsiones semánticas en sus reconstrucciones (Danziger, 2013). El nexo entre el pensamiento crítico y la contextualización social es directo: solo una teoría contextualizada histórica y socialmente se torna completamente comprensible, y solo luego de fundamentarse en la inteligibilidad y comprensión que brinda tal contextualización, el pensamiento puede considerarse crítico por oposición al pensamiento fundamentado en gustos personales, en apelaciones a la autoridad o en simples opiniones (Ennis, 1991; Tao \& Li, 2014a).

En vínculo con esto último surge un tercer argumento acerca del valor de la historia: la incorporación de contenidos históricos a la formación del psicólogo colaboraría con contrarrestar el dogmatismo, la ortodoxia y las peticiones de principio cultivados en el estudiante a partir de su exposición prolongada, durante su socialización universitaria y durante su ejercicio profesional, a talantes doctrinarios y ortodoxos (esto es, irracionalistas, basados en peticiones de principio), susceptibles de ser encarnados por docentes, autores o posturas teóricas específicas, y comunicados explícita o implícitamente a través de contenidos, actitudes, énfasis curriculares, adecuación de los recursos humanos o mecanismos de entrenamiento y supervisión (Danziger, 1994; Roberts, 1982; Vilanova, 1997b). Frente a estas perspectivas que atentan contra el criticismo y el debate público que constituyen características de la ciencia desde una perspectiva sociológica (Klappenbach, 2008/2009; Ziman, 1986), la enseńanza y el aprendizaje acerca de la historia de la psicología, especialmente aquella que incorpora cuestiones historiográficas a sus contenidos, constituye "un instrumento para la metacognición y la toma de conciencia, para la ganancia de control sobre las acciones individuales y colectivas" (Rosa, 2002, p. 212). 
Si la enseñanza de la historia de la psicología efectivamente cuestiona el presente de la disciplina remarcando su contingencia y determinación socio-cultural, cuestionando los asertos de la disciplina en busca de argumentos y evidencias específicas y exponiendo al alumno un panorama plural y multiforme de su ciencia ( $\mathrm{y}$ no un panorama sesgado o tergiversado a partir de preselecciones interesadas), entonces tal enseñanza es susceptible de fomentar ya no solo una modalidad de pensamiento específico (como lo es el pensamiento crítico), sino una modalidad actitudinal específica plural, tolerante y racional (esto es, desafectivizada), con que volverse sobre su propia ciencia y su propio ejercicio profesional. Una enseñanza de este tipo, a la vez que presenta a las escuelas, teorías y autores históricos de la psicología como auténticas y legítimas alternativas (independiente y previamente a cualquier análisis epistemológico en torno a la verdad, eficacia o eficiencia de tales alternativas), colabora con familiarizar al alumno con la raigambre histórica de ciertas temáticas vitales para la psicología contemporánea, como las que refieren a grupos minoritarios y a la geopolítica del conocimiento (Ostrovsky, 2010; Rutherford, 2013; Woodward, 1994). Es en el sentido del contextualismo y del pluralismo así fomentado que Smith (2007) alude a la perspectiva que la historia otorga al psicólogo en formación, y es en dicho sentido que McPherson (1979) destaca que la enseñanza de la historia colabora con limitar el etnocentrismo y el provincialismo que en ciencia constituye uno de los principales causantes del dogmatismo y de la ortodoxia.

Múltiples relevamientos empíricos corroboran estas hipótesis: un ya clásico estudio realizado por Hart y English (1983) concluía que el curso de historia de la psicología en tanto variable situacional modula la elección teórica de los estudiantes. Un análisis conducido por Sánchez, Rivero y Pérez (2000) comprueba un aumento en la complejidad del pensamiento y representación históricos en estudiantes universitarios a partir de la impartición de la asignatura, lo que redundaría en una identidad profesional más coherente y definida. Fernández, Aguinaga, Gallardo y Grassi (2011) indican que la impartición de contenidos históricos en asignaturas introductorias específicas permite el desarrollo de 
competencias generales fundamentales para la redacción y comunicación científica, como lo son la pertinencia, la coherencia y la propiedad conceptual en la escritura académica. Tao y Li han concluido acerca de la existencia de correlación positiva entre la enseñanza de la historia y ciertos cambios en las disposiciones de los alumnos hacia el pensamiento crítico (Tao \& Li, 2014a), y entre la enseñanza de la historia y la motivación de logro (Tao \& Li, 2014b). Esto es coherente con los hallazgos de estudios sobre el impacto de asignaturas sobre historia y filosofía de la ciencia en la mutación y desarrollo de las ideas y perspectivas de los estudiantes respecto a la estructura, dinámica y naturaleza de la ciencia (Abd-El-Khalick \& Lederman, 2000).

\section{El valor de la historia a la luz de la formación de los psicólogos latinoamericanos y rioplatenses}

Las argumentaciones previas, además de tener un valor intrínseco puesto que son producto de las reflexiones de los propios historiadores de la psicología, revelan una gran vigencia y relevancia si viramos hacia el ambiente académico latinoamericano. A partir de las discusiones sobre la formación del psicólogo en los países del Mercosur (Alonso, 1999; Di Doménico, 1996; 1999; García, 2003; 2009b; González \&Rodríguez, 1999; Jacó-Vilela, 1999; Morais Nicaretta, 2009; Salas, 2014) y de Iberoamérica en general (Ardila, 1986; Gallegos, 2009; Toro \& Villegas, 2001; Vilanova, 1993; 1995b), y a la luz del perfil de egresado de psicología propio del Cono Sur de América (Dagfal, 2014; Di Doménico \& Vilanova, 1999; Klappenbach, 2003a; 2003b; 2015; Piacente, 1998), a partir de la década de 1990 se han hecho evidentes en dicha región ciertas carencias formativas y de entrenamiento arraigadas precisamente en los resquicios originados en parte por la deserción respecto de los valores expuestos por las argumentaciones arriba esbozadas.

Si bien es inadecuado generalizar estas carencias a todos los ámbitos académicos latinoamericanos, debe destacarse que en ciertos claustros académicos regionales, como el de la 'Cuenca del Plata' (Argentina, sur de Brasil, Uruguay y Paraguay), estos históricos déficits han sido los rasgos identificatorios de numerosas, cuando no de 
todas, las generaciones de psicólogos profesionales (Di Doménico \& Vilanova, 1999; 2000; Klappenbach, 2004; Vilanova, 1997b), e igualmente han sido reconocidos por académicos no-latinoamericanos que se han interesado en la historia de la psicología regional en su generalidad (Hereford, 1966; Klappenbach, 2014; Marín, Kennedy \& Boyce, 1987). Paralelamente a importantes adelantos científicos y autores notables, definiciones de conjunto sobre la psicología latinoamericana han destacado como rasgos básicamente negativos de la psicología regional hacia el cambio de milenio la escasa originalidad en la producción psicológica — referida la poca teorización original—, el carácter dependiente respecto de producciones exógenas — la adopción predominante de ideas, doctrinas y perspectivas de otras latitudes - y la centración - y limitación voluntaria - en torno al ser humano como objeto de estudio (Ardila, 2004).

De una forma más crítica, se ha apuntado como rasgos de la psicología latinoamericana su mimetismo cientista, su carencia de una epistemología adecuada y un fuerte congelamiento en concepciones dilemáticas y dicotómicas de la disciplina: psicología científica vs. científica espiritualista, humanismo vs. materialismo, psicología reaccionaria vs. psicología progresista (Martín-Baró, 1986). En efecto, el 'dilema olvidado' de la psicología latinoamericana parece haber sido la elección, asunción y seguimiento a rajatabla de uno de los dos metaparadigmas psicológicos de fines del siglo XIX e inicios del siglo XX: la psicología centro-europea de la experiencia inmediata y de la vida interior, y la psicología angloamericana, ambientalista, evolucionista, asociacionista y cuna de la profesión (Vilanova, 1995a). A esto se suma una preferencia por problemas, definiciones y proyecciones aplicadas o técnicas de la psicología, un clima institucional y universitario inestable y debilitado por dictaduras y conflictos sociales —al menos hasta la década de 1990 - y la subsunción de la psicología en conflictos ideológicos que a menudo derivaron en la crítica y resistencia a modelos psicológicos foráneos específicos, especialmente los anglosajones. Estas críticas y resistencias, a menudo basadas en el desconocimiento o la lectura altamente interesada y distorsiva de dichos modelos, en el contexto 
del predominio de orientaciones teóricas y meta-teóricas subjetivistas y filosóficas -el grueso de las carreras de psicología del territorio se crearon en facultades de humanidades, filosofía o letras (Ardila, 1969) y sus docentes fueron mayoritariamente psiquiatras (Alarcón, 2004; Hereford, 1966) - fueron obstáculos en la recepción sistemática de, por caso, el conductismo o el humanismo (Ardila, 1984).

Ciertos escenarios académicos latinoamericanos parecen haber maximizado tales tendencias, especialmente aquellos donde fue común "la escasez de prácticas institucionales, de presupuestos para la investigación y de bibliografía adecuada" (Vilanova, 1987/2003, pág. 16). En este sentido se ha argumentado que desde su inicio la psicología latinoamericana, constituyó un escenario bélico y bipartidista donde la lucha -a grandes rasgos- entre objetivistas y subjetivistas, entre 'cientificistas' y 'humanistas (o, más específicamente, entre conductistas y psicoanalistas) tomó tintes religiosos y herméticos, especialmente (pero no únicamente) en países como Chile, Perú y en la región rioplatense (Arias, 2014; Vilanova, 1995a). Particularmente en Argentina -ejemplar de dichos tintes y de otros que describiremos a continuación-, hacia mediados de siglo XX la conjunción de variados factores dio origen, luego de décadas de investigaciones originales básicas y aplicadas inspiradas en el positivismo y en el evolucionismo, a una formación académica y profesional altamente parcializada y sesgada exclusivamente hacia una de las parcelas del territorio de las psicociencias. Este fenómeno se desarrolló en un marco académico donde lo que dirimía las polémicas eran prácticas verbalistas o la elaboración conceptual y no las demostraciones empíricas, dado que los locus epistémicos, por su inaccesibilidad o inexistencia, rara vez fueron el laboratorio, la investigación y replicación controlada o el consumo crítico de producción internacional de vanguardia (Hereford, 1966; Marin et al., 1987).

Entre otros autores, Vilanova (1987/2003) y Klappenbach (2000c) han argumentado que en Argentina y hacia la fundación de las carreras de grado a partir de 1955, el plantel docente poco idóneo, la pobreza presupuestaria, la excesiva cantidad de alumnos por profesor, la inexistencia de bibliografía adecuada (investigaciones empíricas, evaluadas 
por pares y de carácter científico) y la reducción de las áreas y ámbitos profesionales a favor de la psicología clínica privada y monoteórica especialmente a partir de 1958 fueron algunos de los factores que contribuyeron a configurar el molde (académico pero también cognitivo) que, sin modificaciones sustanciales, dio a luz a cinco décadas de psicólogos argentinos. Dichas deficiencias infraestructurales han sido destacadas para numerosas universidades del territorio (Anónimo, 1987; Courel \& Talak, 2001; Slapak, 1987; Vilanova, 1987). Paralelo a esta dinámica académica e institucional (y con causa necesaria en ella), según Serroni-Copello (1997b) la cultura de los psicólogos argentinos conformada durante dicho medio siglo fue marcadamente precientífica, pretecnológica y predemocrática: la adopción de sistemas teóricos comprehensivistas, de sentido absoluto y de realidades últimas, y su aplicación indiscriminada a toda área extraprofesional, determinó la configuración de un medio ácido para la recepción y formulación sistemática de teorías y metodologías auténticamente científicas (esto es, críticas).

En un país culto, pero propenso a los climas totalitarios y al éxito de discursos de corte dogmático, no fue difícil tomar esos dogmas olvidando el límite y la medida que convienen al científico, y fomentar en cambio una identidad profesional propia de sacerdotes de cultos laicos. (Serroni-Copello, 1997b, p. 65)

Se ha sostenido que la formación académica en psicología en Argentina es monoteórica y dogmática, clinicista (o asistencialista), localista — esto es, aislada de los centros internacionales- y practicista, o tecnicista. Esto es posible a nivel curricular a través de una marcada sustitución de contenidos, donde bajo los rótulos de variados ámbitos y áreas profesionales no clínicas se presentan en esencia conceptos, explicaciones y modalidades claramente clínicas. Finalmente, se ha observado que la psicología argentina es objeto de una fuerte disociación entre la producción investigativa de conocimientos (en ámbitos y foros diseńados para tal fin, que en cualquier caso es y ha sido escasa) y la aplicación tecnológica a nivel de la resolución de problemas concretos (Di Doménico \& Vilanova, 1999; Klappenbach, 2003a; Polanco \& Calabresi, 2011; Serroni-Copello, 1997b; Vilanova, 1995a; 1995b; 
1996; 1997b; Vilanova \& Di Doménico, 1999). A pesar de importantes avances organizacionales e institucionales para revertir tal situación (Di Doménico \& Piacente, 2011; González, 2015b; Klappenbach, 2003a), a la luz de recientes relevamientos y revisiones empíricas, estas tendencias demuestran poco — si algún - auténtico y sustancial retroceso, especialmente en lo tocante a la hegemonía psicoanalítica, a la formación de grado en investigación y al desarrollo de áreas no clínicas (Biglieri et al., 2016; García, 2009a; González \& Dagfal, 2012; González, 2015c; Klappenbach, 2015; Manzo, 2011; Manzo, Fierro y Di Doménico, 2016; Menéndez \& Acosta, 2011; Ostrovsky \& Di Doménico, 2007; Polanco \& Calabresi, 2011; Vázquez Ferrero, 2010; 2016).

En primera instancia, se ha destacado insistentemente la naturaleza monoteórica y dogmática de la enseñanza rioplatense de la psicología. La monoteoría alude al predominio de una postura teórica (aquí, concretamente, el psicoanálisis freudolacaniano) en desmedro de otras teorías y psicopraxiologías, sea como la única teoría impartida en los planes de estudio o, lo que es más factible, como el único sistema teórico y procedimental que se imparte sistemática y transversalmente a lo largo de las carreras de grado. Argentina, en este sentido, ha exacerbado ciertas tendencias del escenario regional a entronizar enfoques y sistemas teóricos conceptuales completos, constituyéndose en un caso tan particular como aislado, si consideramos con Alarcón (2002a; 2004) que si bien el psicoanálisis en Latinoamérica se enseñó "en algunos países con mayor vigor y entusiasmo, [...] no logró posesionarse del espacio académico al punto de desplazar a otras orientaciones teóricas de la psicología. La mayoría de países latinoamericanos apostó por una psicología de orientación científica” (2004, p. 309. Énfasis agregado). En Argentina, como destacan Vilanova y Di Doménico (1999), es típica la retórica "en torno a las benéficas influencias del psicoanálisis sobre los psicólogos que en esta región — por ahora, tan solo— han logrado evadir por su intermedio al positivismo, al experimentalismo, al conductismo y a otros males difundidos por el mundo" (p. 36).

El dogmatismo, con la ortodoxia en su base como mecanismo psicosociológico, alude a la modalidad dependiente y autoritaria de 
apropiación, reapropiación y defensa de teorías científicas, y en general de la totalidad del resto del conocimiento. En Argentina, y por lo descrito arriba, este dogmatismo se encastra mayoritariamente con la impartición del psicoanálisis (Vainer, 1997). Semejante a la dinámica de prácticas religiosas y sectarias (Deconchy, 1984; Tosi \& Tosi, 2014), el conocimiento científico se reduce al saber laxamente definido, y este último, a su vez, lejos de surgir del consenso a partir del debate racional y argumentado, es "algo que se recoge de los libros ya escritos, o se escucha de labios autorizados, o se capta por aprendizajes iniciáticos" (Vilanova, 1996, p. 206). En este sentido, y a nivel de la formación de grado, se deserta la investigación empírica, el pluralismo teórico y el escepticismo razonado (corazón de la empresa científica) a favor de la apelación a alguna autoridad iluminada cuyas exégesis de obras necesariamente caducas constituyen el reemplazo a la investigación sistemática, pública y revisada por pares (Vorano, Tosi \& Tosi, 2016).

Así, los problemas empíricos, cuando no son anulados o recodificados en términos de tales exégesis, buscan solucionarse a partir de la indagación del pasado (es decir, retrospectivamente), y no a través de la investigación científica novedosa o del escrutinio de la producción psicológica contemporánea. Es sugerente el aislamiento de Argentina respecto del escenario regional si se emprende una comparación entre la investigación científica e institucionalizada realizada en Argentina (especialmente a partir de 1955) y aquella realizada en el territorio, especialmente multiforme y heterogénea (Alarcón, 2002c).

Estos problemas cobran un cariz diverso en el contexto de los sentidos de la historia descritos en el apartado anterior. A pesar de que las cuestiones sobre la ubicación, la naturaleza y el enfoque historiográfico idóneo del curso de historia de la psicología constituyen debates abiertos (Rutherford \& Pickren, 2015), si se considera la reiterada observación de la enseńanza de la historia de la psicología debería ser plural y debería abarcar una diversidad marcada de escuelas, enfoques y sistemas (Dagenbach, 1999; Pickren, 2012; Smith, 1982; Vilanova, 2000), la monoteoría se revela a la luz de tal pluralidad como un imperativo irracional y fundamentado en intereses espurios. Aún más, si se considera 
el énfasis de la historia de la psicología en el trabajo crítico y no presentista sobre fuentes primarias y en la contextualización social de teorías y autores más allá del 'gran genio aislado' (Furumoto, 1989; Robinson, 1979; Rutherford, 2014), la historia de la ciencia expone la distorsión en que necesariamente incurre todo exégeta cuando intenta justificar el presente de su doctrina, o reducir el campo total de una disciplina como lo es la psicología, a través del supuesto sentido que brota de las lecturas de los textos del fundador aislado en calidad de 'genio creativo' ex nibilo.

Adicionalmente, contra la petición de principio y la suspensión de juicio crítico que requiere una formación universitaria dogmática, la enseñanza de la historia de la psicología expone al alumno a "la fluidez y la relatividad del saber científico y las mudables razones sociales que subyacen a la creación de profesiones" (Vilanova, 1997a, p. 21; van Strien, 1990). En el mismo sentido, los autores latinoamericanos defienden, tal como lo hicieran autores europeos y norteamericanos, que la enseñanza de la historia "[promueve] el desapego emocional hacia las doctrinas y [desalienta] las actitudes creenciales" (Vilanova, 1997a, p. 22; Vezzetti, 2007). En este punto, la historia social de la ciencia (es decir, la que recurre a factores sociológicos, culturales, institucionales y políticos para contextualizar y articular sus narrativas) constituye uno de los enfoques historiográficos que permite que los acontecimientos y sucesos de la psicología se ubiquen en una perspectiva más amplia (Rutherford, 2014) y que simultáneamente eludan los presentismos, teleologismos e idealismos de ciertas tendencias intelectualistas en historia de la ciencia (Danziger, 2013; Valdés-Fauly \& de la Torre Molina, 1985; Vilanova, 2000).

Continuando con las peculiaridades de la psicología argentina en el contexto de las psicociencias latinoamericanas, se ha destacado que la formación rioplatense es eminentemente clinicista y, adicionalmente, practicista (Di Doménico \& Vilanova, 1999; 2000; Klappenbach, 2003b; Vilanova, 1996). Estas características, regulares en el grueso de la psicología latinoamericana (Dembo, 1993; Rojo González, 1993), han sido alimentadas por las peculiaridades de la formación históricamente impartida en las carreras. Lo primero alude esencialmente a la 
reducción de las áreas de desempeño profesional del psicólogo al ámbito clínico (reducción que, a la luz de la monoteoría y el dogmatismo antes descritos, desemboca entre otras cosas en la reducción de la psicología al psicoanálisis clínico, freudiano o lacaniano). El practicismo alude a la deserción de la investigación como la vía regia para la producción de conocimiento en pos del 'ojo clínico' (es decir, el cumulo de experiencia clínica privada e inescrutable por pares), de la inventiva personal o de la inspiración literaria. Esta deserción implica necesariamente confundir teoría y técnica, conocimiento objetivo provisorio y certeza subjetiva inconmovible, e investigación (y corroboración) de conocimiento y aplicación (no controlada) de conjeturas individuales. En términos de Vilanova (1996), esto implica que el graduado "rechaza la investigación incluso en los estudios de master o de doctor y ostenta una actitud admirativa ante las técnicas, el 'ojo clínico', la 'experiencia' y otras vías artesanales de acceso a los hechos" (p. 207).

Por otro lado, esto implica un plantel docente en los ciclos profesionales de las carreras que no reconocen "ninguna relación entre el servicio para el que capacitan y la investigación [...], [este error] nacido en la identificación del actuar profesional con un tipo de investigación sui generis [y en la ignorancia de] que las prácticas no son autoconvalidadoras" (Vilanova, 1996, p. 207), y conlleva al borramiento de la investigación aplicada (es decir, de los efectos e intervenciones profesionales) de la formación de grado. En este sentido, países latinoamericanos como Argentina han hipostasiado la tendencia aplicada de la psicología latinoamericana, desembocando en verdaderos profesionalismos que conciben al psicólogo como un tecnólogo aplicado, especialmente en el área de la salud, más que como un científico básico con intereses transversales en todas las subdisciplinas psicológicas.

Agregados todos estos factores, el común de la psicología argentina (al menos tal como es comunicada en el grueso de los curricula) parece reducirse al ejercicio acrítico y no controlado metodológica o profesionalmente de una psicopraxiología clínica monolítica, omniexplicativa (es decir, erigida como fuente de problemas y respuestas aplicables a otros ámbitos disciplinares) desconocedora de sus propios 
sesgos y limitaciones, autolegitimada solo al precio de anular o retraducir otras perspectivas, prácticamente incontrastable — por sus enunciados extremadamente generales, vagos, inefables y surgidos de experiencias clínicas anecdóticas, privadas e inescrutables - y en desmedro de otras legítimas alternativas. ${ }^{3}$ Tal ejercicio profesional tiene su origen directo en el modelo profesional transmitido por las universidades, donde es la psicología clínica (en los términos recién descritos) el ámbito del que parten los principios explicativos y las líneas directrices, los que a su vez tienden a reemplazar los contenidos y lineamientos de las demás áreas y ámbitos profesionales (Klappenbach, 2003a; Vilanova, 1996).

Situar históricamente este estado actual, en el desarrollo de la psicología en el país y en el contexto de las diferencias entre Argentina y la psicología de otros países latinoamericanos en un sentido comparativo, constituye una de las formas en que la historia de la psicología contribuye críticamente con la formación de los psicólogos: esto es, al concebir la propia formación académica y sus bases institucionales como un fenómeno contingente que tiene raíces sociales, culturales y políticas específicas (Gallegos, 2016).

A partir de una enseńanza de la historia de la psicología plural, crítica, y no legitimante de ciertas escuelas o sistemas específicos, pueden revisarse y discutirse clara y fundamentadamente todos los principios que sostienen tal formación rioplatense contemporánea, cumpliéndose la máxima de que la historia permite cuestionar en perspectiva el estado actual de la disciplina (Smith, 2016). En primera instancia, puede rebatirse la reducción de la psicología a la psicología clínica al evidenciar la preexistencia cronológica y teórica de la primera, que solo en un segundo momento se proyectó en un campo técnico-praxiológico o profesional como el clínico (van Hoorn, 1984). Esto ofrece al alumno un panorama disciplinar que expone una gran variedad de campos teóricos y empíricos, y dentro de los cuales la clínica es solo una componente aplicada del campo total de la psicología, incluso la latinoamericana (García, 2014). De igual forma, la reducción de la 
psicología clínica al psicoanálisis puede evidenciarse como ilegítima a partir de instruir acerca de los orígenes, decursos y resultados disímiles de ambos campos (Benjamin, 2005; Vilanova, 1990).

En segunda instancia, una historia de la psicología que incorpore cuestiones epistemológicas y metateóricas es capaz de exponer al alumno la clara distinción entre una teoría básica, una tecnología y una psicopraxiología (Serroni-Copello, 1997a), con las implicancias teóricas de tal distinción, y en la medida en que tal distinción pueda detectarse en las fuentes primarias (evitándose así una distorsión de las evidencias históricas). En tercera instancia, la identificación entre una teoría o sistema psicológico (tal como el psicoanálisis) y una ciencia y una profesión como la psicología es claramente rebatible a partir tanto del análisis histórico del origen, desarrollo y profesionalización de la segunda, como del redimensionamiento de cada teoría, constructo y problemática psicológica en función de las circunstancias socio-históricas particulares de que han emergido (Dagfal, 2015; Danziger, 1984; van Strien, 1990). Este importante redimensionamiento colaboraría con reintroducir el carácter inherentemente histórico e hipotético a los constructos psicológicos que son materia de estudio y de pesquisa de los psicólogos (Danziger, 2001; 2013), y permitiría ubicar a priori y a pie de igualdad a todas las 'escuelas' o sistemas psicológicos históricos de la disciplina. Esto es especialmente necesario en Argentina dado el naturalismo, el universalismo y el ahistoricismo con que los profesionales rioplatenses conciben ciertos constructos que se recuperan directamente (y sin mediar crítica alguna) de textos freudianos centenarios; constructos que, como el de 'inconciente freudiano', 'pulsión' o 'complejo de Edipo', en definitiva constituyen propuestas hipotéticas (Piacente, 1998). Respecto de la carencia de evidencia empírica sistemática sobre tales constructos (al menos tal y como se definen, imparten y blanden en las academias argentinas), la historia realmente constituye una fuente idónea de perspectiva crítica: como afirma Smith (2016),

Freud le dio un lugar tan seminal a la historia en su propagación del psicoanálisis que dicha historia se volvió una parte de la verdad pretendida por el campo. Como consecuencia, la defensa o la crítica 
del contenido o las prácticas del psicoanálisis se han anudado, desde el comienzo, a lo que se ha dicho sobre la creación por Freud del campo en primer lugar $[\ldots]$ al traer a la luz la arbitrariedad detrás de las narrativas y de las interpretaciones de los hechos realizadas por Freud, el estudio histórico relativiza y deslegitima la teoría del psicoanálisis de una forma mucho más efectiva que cualquier crítica epistemológica. (pp. 13-14)

En cuarta instancia, la historia introduce al estudio de lo que consensuadamente se concibe como el rol profesional del psicólogo, al evidenciar los genuinos problemas empíricos que, heredados del pasado, continúan irresueltos y demandantes de los esfuerzos de la comunidad científica. En línea con esto, se sostiene que la historia debe "articular conceptualmente el ciclo básico, abocado a la producción de conocimiento, con el ciclo profesional, destinado a las aplicaciones y a los servicios sociales. Solo el desarrollo histórico de la psicología explica esta 'doble formación' que el alumno recibe" (Vilanova, 1997a, p. 22). Finalmente, una historia historicista (es decir, que intente recuperar el pasado en términos del pasado mismo minimizando la distorsión interesada de las propuestas históricas de la disciplina, y minimizando al máximo el whiggismo) pondría en evidencia la ilegitimidad en que incurre todo intento de retraducción o reducción de una (o varias) perspectivas psicológicas como consecuencia de la asunción de un sistema entronizado y concebido como auténtica y genuinamente verdadero.

$\mathrm{Si}$ adicionalmente consideramos que el estado de la psicología argentina se debe, entre otras razones y en lo que respecta a la formación, a la existencia de énfasis curriculares (Roberts, 1982) nocivos para la identidad profesional del psicólogo —es decir, a la existencia de mensajes implícitos y explícitos en la formación universitaria que desalientan entre otras cosas la consideración del conocimiento empírico consensuado, el recurso a la investigación internacional actualizada o la asunción de una concepción realista-crítica de la ciencia y sus objetos-; si consideramos, en efecto, la existencia de tales énfasis curriculares, entonces la enseñanza de la historia de la psicología desde una perspectiva plural, crítica y no dogmática habilita posibles énfasis curriculares alternativos. Tal enseñanza debería acentuar, entre otras 
cosas, la naturaleza siempre mutable — mas no absolutamente relativa- del conocimiento científico, incluso el histórico (Loredo, 1998), remarcando el carácter imprescindible de la investigación controlada, abierta y pública como respuesta a los problemas de la psicología, y destacando el recurso a la argumentación y al debate por sobre el recurso a las pasiones y gustos personales en los foros estudiantiles, académicos y científicos. En definitiva, la enseñanza de una historia crítica, social, contextual y plural de la psicología fomenta en los alumnos el 'talante crítico' (Rosa, 2002), informado y autorreflexivo, que no es otro que el talante científico (Benjamin, 2010; Fierro \& Di Doménico, 2017).

\section{La historia de la psicología latinoamericana en Argentina: relevamiento del estado actual de su enseñanza}

En el contexto de la relevancia pedagógica de la historiografía en América Latina, y con el objetivo de caracterizar el estado actual de la enseñanza de la historia regional de la psicología en Argentina, se describe a continuación un análisis cuantitativo y cualitativo sobre asignaturas históricas en carreras de grado de psicología del país.

\section{Método}

El presente relevamiento y análisis constituye un análisis descriptivo de tipo sociobibliométrico, de diseńo ex post facto retrospectivo, de acuerdo a relevamientos anteriores (Klappenbach, 2008/2009; Marińelarena-Dondena $\&$ Klappenbach, 2016) y a clasificaciones de estudios empíricos en psicología (Montero \& León, 2007).

\section{Participantes}

Fueron relevadas y analizadas en calidad de contenidos las referencias bibliográficas obligatorias y ampliatorias $(n=798)$ de los Programas de Trabajo Docente (sílabos) vigentes entre 2011 y 2014 de ocho asignaturas sobre contenidos históricos de la psicología al interior de siete facultades de psicología de universidades nacionales de gestión pública argentinas. 
La elección de universidades de gestión pública se justifica dado que tales casas de estudio nacionales alojaban hacia el 2015 aproximadamente al 51,3\% de estudiantes de psicología activos en el territorio (49,455 estudiantes) y al 80,4\% del total de estudiantes de psicología en universidades nacionales de gestión pública (Alonso \& Klinar, 2016). Complementariamente, tales unidades académicas han producido históricamente el $64,5 \%$ del total de egresados desde la profesionalización de la psicología en Argentina. La Tabla 1 presenta información sobre matrícula, distribución de alumnos y docentes disponibles en los cursos relevados en función de las universidades seleccionadas.

\section{Procedimiento}

Se realizó un relevamiento y análisis documental, de tipo sociobibliométrico, de las referencias totales (obligatorias y ampliatorias) de los programas arriba especificados. El análisis bibliométrico aplicado a la psicología goza de una considerable y consolidada tradición (Klappenbach, 2008/2009; Miguel \& Dimitri, 2013), especialmente en su aplicación a la historia de la psicología (Carpintero \& Peiró, 1981; Carpintero \& Tortosa, 1990) y a la historia de las prácticas locales en psicología (Jaraba Barrios, Guerrero-Castro, Gomez Morales \& López López, 2011). En el contexto de concebir a la formación universitaria en psicología como una práctica de renovación y retroalimentación del campo socioprofesional, la socio-bibliometría es considerada por múltiples autores (Fierro, en prensa; Klappenbach, 2013; Vázquez Ferrero, 2010; Vázquez-Ferrero \& Colombo, 2008) como especialmente apta para evaluar los contenidos de la currícula y los perfiles profesionales que estas encausan.

Cada referencia bibliográfica relevada se analizó y clasificó de acuerdo a ciertos indicadores bibliométricos propuestos por estudios sobre estudios cuantitativos sobre formación en psicología (Klappenbach, 2013) y enseñanza de la historia (Visca \& Moya, 2013): a saber, el autor de la referencia, el año de publicación, la nacionalidad del autor, el tipo o carácter de la fuente (primaria, secundaria o terciaria), el tipo de edición (libro, capítulo de libro, publicación de 
Historia de la psicología y formación de psicólogos argentinos... / Fierro

\section{Tabla 1}

Cursos argentinos de Historia de la Psicología en universidades públicas en relación con variables infraestructurales, 2011-2014

\begin{tabular}{|c|c|c|c|c|c|}
\hline Universidad & $\begin{array}{c}\text { Matrícula } \\
\text { total activa } \\
\text { hacia el } \\
2015^{*}\end{array}$ & $\begin{array}{l}\text { Porcentaje } \\
\text { de matrícula } \\
\text { en relación } \\
\text { al total de } \\
\text { alumnos en } \\
\text { universidades } \\
\text { nacionales }\end{array}$ & $\begin{array}{l}\text { Cantidad } \\
\text { de } \\
\text { ingresantes } \\
\text { en } 2015^{* *}\end{array}$ & $\begin{array}{l}\text { Número de } \\
\text { auxiliares } \\
\text { docentes en } \\
\text { los cursos de } \\
\text { historia }\end{array}$ & $\begin{array}{l}\text { Cantidad } \\
\text { de alumnos } \\
\text { por auxiliar }\end{array}$ \\
\hline $\begin{array}{l}\text { Nacional de } \\
\text { La Plata }\end{array}$ & 12743 & $20.7 \%$ & 1465 & 10 auxiliares & 146 \\
\hline Buenos Aires & 11827 & $19.2 \%$ & 2320 & 19 auxiliares & 122 \\
\hline $\begin{array}{l}\text { Nacional de } \\
\text { Córdoba }\end{array}$ & 10755 & $17.4 \%$ & 2837 & 7 auxiliares & 405 \\
\hline $\begin{array}{l}\text { Nacional de } \\
\text { Comahue }\end{array}$ & 8447 & $13.7 \%$ & 451 & 4 auxiliares & 113 \\
\hline $\begin{array}{l}\text { Nacional de } \\
\text { Tucumán }\end{array}$ & 5534 & $9 \%$ & 997 & $\begin{array}{l}2 \text { titulares- } \\
\text { auxiliares }\end{array}$ & $499^{* * *}$ \\
\hline $\begin{array}{l}\text { Nacional de } \\
\text { Mar del Plata }\end{array}$ & 3719 & $6 \%$ & 936 & 7 auxiliares & 133 \\
\hline $\begin{array}{l}\text { Nacional de } \\
\text { San Luis }\end{array}$ & 1444 & $2.8 \%$ & 231 & 3 auxiliares & 77 \\
\hline
\end{tabular}

"Según el relevamiento nacional de Alonso y Klinar (2016).

"Considerando los relevamientos cuantitativos previos, la cantidad de ingresantes a carreras de psicología en el país ha aumentado progresivamente. Por tanto, estas cantidades no pueden considerarse excepciones o 'picos' sino la norma en un sentido estricto.

"** Dado que el curso se ofrece cuatrimestralmente y cada una de las mitades de los estudiantes cursa la asignatura en uno de esos cuatrimestres, técnicamente la mitad de la proporción expuesta representa el número de estudiantes por auxiliar por semestre (249 estudiantes por cada auxiliar). 
revista periódica, etc.), el idioma, el género y la nacionalidad del autor. Las referencias bibliográficas fueron analizadas a través de estadística descriptiva, en términos de frecuencias y porcentajes absolutos y relativos con la finalidad de delinear posibles líneas transversales y núcleos comunes de contenido en las asignaturas. Estos indicadores permiten obtener información sobre aspectos centrales que a la luz de lo discutido hasta aquí informan sobre la contribución de los cursos de historia de la psicología a los aspectos problemáticos de la formación del psicólogo, como lo son el relegamiento de la investigación, el reduccionismo monoteórico y el pluralismo disciplinar, entre otros.

Los resultados obtenidos fueron analizados en el contexto de la argumentación expuesta en las secciones anteriores, especialmente en lo referente al pluralismo historiográfico, a la importancia de una presentación heterogénea y diversa de la psicología y al fomento del pensamiento crítico para la psicología latinoamericana.

\section{Resultados}

El análisis sociobibliométrico arroja un núcleo común pero circunscripto de autores y títulos, compartido horizontalmente entre varias asignaturas. La tabla 1 expone tal núcleo a partir de las obras referenciadas tres o más veces en los programas (menor frecuencia de citación que permite inferir con probabilidad la presencia de un título en dos asignaturas). Estos títulos comportan solo el 10,3\% ( $\mathrm{N}=82)$ del total de las referencias. Más allá de este núcleo común, existe una gran dispersión de contenidos entre las asignaturas.

A su vez, se evidencia una incorporación comparativamente reducida de investigaciones contemporáneas en historia de la psicología. Como expone la tabla 2, un 25,5\% del total de las referencias corresponden a publicaciones periódicas en revistas científicas con referato (es decir, papers en journals). De dichas referencias, menos del 10\% refiere a revistas específicas sobre temáticas históricas o historiográficas. La tabla 4 especifica las revistas incluidas en la bibliografía, resaltándose 
aquellas específicas a historia e historiografía de la ciencia. La media de publicación de artículos de revistas científicas es el año 1996, y el $45,5 \%$ de dichos artículos es previo al año 2000. Los autores más citados en artículos de revistas incluyen a psicólogos-historiadores argentinos que son o han sido docentes en asignaturas de historia (como Hugo Klappenbach, Hugo Vezzetti, Alejandro Dagfal y Alberto Vilanova, entre otros) y, en menor medida, a historiadores internacionales (como Kurt Danziger, Allan Buss y Antonio Caparrós). Varios de los autores argentinos son los productores más visibles de la historiografía iberoamericana (Carpintero, 2007).

Finalmente, el $70,8 \%$ de las referencias totales remite a fuentes secundarias, y solo el 22,6\% remite a fuentes primarias o históricas. $\mathrm{Si}$ se agregan los resultados sobre las fuentes secundarias y sobre la incorporación de investigaciones históricas, entonces se hipotetiza que la mayor parte de las fuentes secundarias no-históricas no serían producciones actualizadas o de reciente publicación, sino producciones longevas debido a la propia dinámica tanto de la producción científica como de la investigación histórica en psicología. La longevidad se corrobora al analizar los tipos de fuente expuestos en la tabla 2: las fuentes secundarias que constituyen capítulos de libro de único autor comprenden casi un quinto del total de referencias y registran una media de año de publicación de 1994, y los libros completos de fuente secundaria que comprenden poco más de un quinto de las referencias totales registran una media de año de publicación de 1993. Sin desagregarse en tipos de edición, la media de ańo de publicación de las fuentes secundarias es de 1999.

Respecto a la nacionalidad de los autores, existe un claro predominio de autores argentinos por sobre otras nacionalidades, tal como lo demuestra la tabla 3. En lo que interesa a nuestros objetivos, existe una escasa representación de autores latinoamericanos no-argentinos. Con excepción de la cátedra II de la Universidad de Buenos Aires, la cátedra de la Universidad Nacional de La Plata y la cátedra de la Universidad Nacional del Comahue, las asignaturas registran un predominio de bibliografía de autores argentinos, lo cual es coincidente con estudios 
previos (Fierro et al., 2017). Finalmente, los autores de género masculino comprenden un $78,8 \%$ de las referencias totales, contra un $12,3 \%$ de autores de género femenino y un $8,9 \%$ de referencias a obras cuyos autores y co-autores comprenden ambos sexos.

\section{Tabla 2}

Tipos de edición referenciados con frecuencia de citación mayor a uno en los programas de historia de la psicología

\begin{tabular}{lccc}
\hline Tipo de edición & Frecuencia & Porcentaje & $\begin{array}{c}\text { Porcentaje } \\
\text { acumulado }\end{array}$ \\
\hline Capítulo de libro & 260 & 32.6 & 32.6 \\
Artículo de publicación periódica & 203 & 25.5 & 58.1 \\
Libro & 166 & 20.8 & 78.9 \\
Capítulo de libro en libro colectivo & 59 & 7.4 & 86.3 \\
editado & & 3.5 & 89.8 \\
Ficha de cátedra & 28 & 2.8 & 92.6 \\
Artículo en obras completas & 22 & & \\
Capítulo de libro en libro colectivo & 17 & 2.1 & 94.7 \\
compilado & 10 & 1.3 & 96.0 \\
Libro colectivo editado & 7 & .9 & 96.9 \\
Libro colectivo compilado & 5 & .6 & 97.5 \\
Actas de congreso & 3 & .4 & 97.9 \\
Artículo de periódico & 3 & .4 & 98.2 \\
Otro (memoria de congreso) & 2 & .3 & 98.5 \\
Otros (mímeo) & 2 & .3 & 98.7 \\
Otros (tesis doctoral) & & & \\
\hline
\end{tabular}




\section{Tabla 3}

Titulos con frecuencia de citación mayor a dos en los contenidos de las asignaturas de historia de la psicología

\begin{tabular}{|c|c|c|c|}
\hline Título & Autor & Frec. & $\begin{array}{l}\text { Porc. } \\
\text { acum. }\end{array}$ \\
\hline Freud en las Pampas & M. Ben Plotkin & 5 & 1.6 \\
\hline Una historia crítica de la psicología & N. Rose & 5 & 1.3 \\
\hline $\begin{array}{l}\text { Historias de la psicología: problemas, funciones y } \\
\text { objetivos }\end{array}$ & H. Vezzetti & 4 & 1.8 \\
\hline $\begin{array}{l}\text { Inventing our selves. Psychology, Power and } \\
\text { Personhood }\end{array}$ & N. Rose & 4 & 2.3 \\
\hline Los orígenes sociales de la psicología moderna & K. Danziger & 4 & 2.8 \\
\hline Aventuras de Freud en el país de los argentinos & H. Vezzetti & 4 & 3.1 \\
\hline $\begin{array}{l}\text { Crisis de la psicología: ¿Singular o plural? Aproxi- } \\
\text { mación a algo más que un concepto historiográfico }\end{array}$ & A. Caparrós & 3 & 3.5 \\
\hline $\begin{array}{l}\text { Del tratamiento moral a las psicoterapias. Observa- } \\
\text { ciones sobre la formación de la idea contemporánea } \\
\text { de psicoterapia }\end{array}$ & G. Swain & 3 & 3.9 \\
\hline El título profesional de psicólogo en Argentina & H. Klappenbach & 3 & 4.3 \\
\hline Antecedentes históricos y situación actual & H. Klappenbach & & \\
\hline El verdadero Charcot & $\begin{array}{l}\text { M. Gauchet y } \\
\text { G. Swain }\end{array}$ & 3 & 4.6 \\
\hline Estructura social de la experimentación en Psicología & K. Danziger & 3 & 5.0 \\
\hline $\begin{array}{l}\text { Galton, el nacimiento de la psicología diferencial y la } \\
\text { eugenesia: factores sociales, políticos y económicos }\end{array}$ & A. Buss & 3 & 5.4 \\
\hline $\begin{array}{l}\text { Hacia un marco conceptual para una historia crítica } \\
\text { de la psicología }\end{array}$ & K. Danziger & 3 & 5.8 \\
\hline Introducción a Compendio de Psicología & W. Wundt & 3 & 6.1 \\
\hline $\begin{array}{l}\text { José Bleger y los inicios de una psicología psicoanalí- } \\
\text { tica en la Argentina de los } 60\end{array}$ & A. Dagfal & 3 & 6.5 \\
\hline $\begin{array}{l}\text { La batalla de cien años. Historia del psicoanálisis en } \\
\text { Francia Vol. } 1 \text { (1885-1939) }\end{array}$ & E. Roudinesco & 3 & 6.9 \\
\hline La falsa medida del hombre & S. Jay Gould & 3 & 7.3 \\
\hline La historia de la introspección revisada & K. Danziger & 3 & 7.7 \\
\hline Las Psicologías del fin de siglo a la luz de su historia & H. Vezzetti & 3 & 8.0 \\
\hline Las raíces históricas del laboratorio psicológico & K. Danziger & 3 & 8.4 \\
\hline $\begin{array}{l}\text { Los anormales. Curso en el Collège de France } \\
(1974-1975)\end{array}$ & M. Foucault & 3 & 8.8 \\
\hline $\begin{array}{l}\text { Los comienzos de la psicología como disciplina } \\
\text { universitaria y profesional: debates, herencias, pro- } \\
\text { yecciones sobre la sociedad }\end{array}$ & H. Vezzetti & 3 & 9.2 \\
\hline Mental Health and World Citizenship & J. R. Rees & 3 & 9.5 \\
\hline
\end{tabular}




\begin{tabular}{lccc} 
Título & Autor & Frec. & $\begin{array}{c}\text { Porc. } \\
\text { acum. }\end{array}$ \\
\hline $\begin{array}{l}\text { Periodización de la Psicología en Argentina } \\
\begin{array}{l}\text { Progreso, degeneración y darwinismo en la primera } \\
\text { psicología argentina, 1900-1920 }\end{array}\end{array}$ & $\begin{array}{l}\text { A. Tappenbach } \\
\text { A. Talak }\end{array}$ & 3 & 9.9 \\
\hline
\end{tabular}

En su conjunto, factores como la nacionalidad de los autores y la prevalencia de textos de ciertos historiadores argentinos parecen explicar a su vez cierto patrón de incorporación de literatura en las asignaturas priorizando la producción de los docentes a cargo de cada asignatura. Ciertas asignaturas parecen tener un patrón de citación de acuerdo al cual la bibliografía de los cursos remite predominantemente a trabajos cuyo autor es el docente que actual o históricamente ha obrado como titular o responsable de cada curso, mientras que otras asignaturas no parecen guiarse por tal criterio. Esto, que resulta natural por la filiación de los investigadores a sus cátedras respectivas, no permite sin embargo explicar por qué en ciertas asignaturas, a la vez que se orientan según tal criterio, la referencia a trabajos de titulares a cargo de otras asignaturas históricas es escasa o nula. Las Figuras 1 y 2 exponen los datos respecto a este fenómeno.

\section{Tabla 4}

Publicaciones periódicas con frecuencia de citación mayor a 1 en los programas de historia de la psicología

Nombre de publicación periódica

Frecuencia Porcentaje $\begin{gathered}\text { Porc. } \\ \text { acumulado }\end{gathered}$

Revista de Historia de la Psicología

24

11.8

11.8

Cuadernos Argentinos de Historia de la Psicología

Journal of the History of the Behavioral Sciences

American Psychologist

Acta Psiquiátrica y Psicológica de América Latina

Revista Latinoamericana de Psicología

Anuario de Psicología

Cátedra I Historia de la Psicología Facultad de Psi- 
Nombre de publicación periódica

Frecuencia Porcentaje

Porc. acumulado

History of Psychology

$\begin{array}{ll}2.5 & 39.9\end{array}$

History of the Human Sciences

2.5

42.4

Revista Universitaria de Psicoanálisis

Anuario de Investigaciones

Theory \& Psychology

2.5

44.8

Cátedra Psicología I Facultad de Psicología UNLP

Facultad de Psicología UBA

$2.0 \quad 46.8$

Revista de la Asociación Española de

2.0

48.8

Neuropsiquiatría

Revista de Psicología General y Aplicada

1.5

50.2

Annales Médico Psychologiques

Anuario Investigaciones de la Facultad de Psicología

UBA

Cuadernos de Psicología Concreta

Departamento de Publicaciones de la Facultad de

Psicología de la UBA

Diálogos. Revista Científica de Psicología, Ciencias

Sociales, Humanidades y Ciencias de la Salud

Fundamentos en Humanidades

Journal of Russian and East European Psychology

Journal of the History of Medicine

1.0

L'Évolution psychiatrique

1.0

60.6

Memorándum

1.0

61.6

Psicología em Estudo

1.0

62.6

Psychological Bulletin

1.0

63.5

Psychological Review

1.0

Revista Argentina de Psicología

1.0

65.5

Revista Cubana de Psicología

1.0

66.5

Teaching of Psychology

1.0

67.5

The American Journal of Psychiatry

1.0

68.5

1.0

69.5 


\section{Discusión}

\section{Elevada dispersión y heterogeneidad de autores y titulos}

La considerable dispersión de autores y títulos (solo contrarrestada por los circunscriptos contenidos transversalmente compartidos, expuestos en la tabla 2) indicaría una elevada heterogeneidad respecto de la enseñanza de la historia de la psicología en el país.

A esta heterogeneidad de contenidos se contrapone la aceptación declarativa del 'enfoque crítico' en historia de la psicología por parte de todas las asignaturas, especialmente recepcionado por los historiadores locales desde la década de 1990 (Sanz, 1997). Sin embargo, el grado y carácter de esta adscripción es variable, puesto que los autores y obras con que se identifica este enfoque son múltiples y en ocasiones disímiles entre sí (la 'escuela de York', la historiografía marxista, K. Danziger, N. Rose, H. Vezzetti, M. Foucault o G. Canguilhem, entre otros). Esto permitiría concluir que, en línea con la libertad de cátedra detentada por las asignaturas, existe poco consenso entre las cátedras respecto a los contenidos centrales que se identificarían con la educación histórica de los psicólogos.

\section{Incorporación limitada de investigaciones y producciones históricas actualizadas}

La comparativamente baja frecuencia de investigaciones históricas actualizadas previamente constatada para la bibliografía obligatoria de las asignaturas (Visca \& Moya, 2013) se corrobora en este estudio para la bibliografía completa de los sílabos. Esto constituye un desafío para la instrucción histórica que pretenda fomentar la investigación (histórica o de otro tipo) como motor disciplinar y como destino profesional de los graduados. La inclusión de referencias bibliográficas a trabajos actualizados provenientes de publicaciones periódicas, especialmente de revistas temáticas sobre la subdisciplina, es necesaria para exponer a la Historia de la Psicología como un campo disciplinar relevante y en constante actualización, y para la presentación de la historia de la 
psicología como un objeto de estudio dinámico, susceptible de revisión y que alcanza efectivamente el presente (Benjamin, 2008). Esto colaboraría tanto con presentar a la historia de la psicología como un ámbito legítimo de investigación empírica, tan activo y riguroso a nivel internacional como los demás campos empíricos de la psicología, como con minar el practicismo y el tecnicismo hegemónico en los psicólogos argentinos, al instruir acerca de un posible ámbito de desempeño académico e investigativo legítimamente alternativo y complementario a los ámbitos profesionales de la psicología estrictamente definidos. Enmarcada la Historia de la Psicología en el ciclo de formación básica —auténtico fundamento del ejercicio profesional—, su estudio factiblemente minorizaría la perseveración de aquellos sesgos, toda vez que la historia, en tanto que actividad académica que requiere de recolección, análisis y publicación sistemática de datos y resultados, puede comunicar la idea —difícilmente whiggista en la actualidad — de que "quedó atrás, y es historia, la psicología especulativa y con ella el antiguo prejuicio sobre la imposibilidad de cuantificar, explicar y predecir el comportamiento humano" (Alarcón, 2002b, p. 212). En tal sentido, un análisis crítico de la historia permite confinar al pasado gran parte de los 'dilemas' o de las 'dicotomías' de la psicología argentina, al menos en el formato en que se blanden desde hace décadas (Vilanova, 1995a).

En el mismo sentido se justificaría incorporación de más producciones históricas contemporáneas en la enseńanza de la historia. Reubicando a la historia de la psicología (y con ella, a la epistemología y a la filosofía de la psicología) como fuente de problemas legítimos y actuales, las propias psicopraxiologías (clínicas, laborales, comunitarias, entre otras) necesariamente desaparecen como los únicos y 'verdaderos' espacios problematizadores en la disciplina, tal como aparecen en los curricula argentinos. En una redefinición tal de los énfasis curriculares, la historia abandonaría su actual carácter de contenido casi suntuario (Vilanova, 2000), reasumiendo su lugar de subdisciplina al interior de la psicología y de la historia de la ciencia. 


\section{Predominio de autores argentinos y sus implicancias}

La producción historiográfica indica que los historiadores de la psicología suelen historiar sobre la psicología de su propio país (Klappenbach \& Jacó-Vilela, 2016; Klappenbach \& Fierro, 2016). A la vez, la historiografía de la psicología iberoamericana suele ser comunicada y difundida por autores iberoamericanos (Carpintero, 2007). Considerado esto, el amplio predominio de autores argentinos en la bibliografía, expuesto en la tabla 5, indicaría que en los sílabos se identifica mayoritariamente la historia de la disciplina a nivel internacional con la historia de los avatares y perspectivas de la psicología local. Este patrón de ausencia significativa de autores latinoamericanos no argentinos en las asignaturas de historia es problemático puesto que parece seguir un patrón más general: hacia 2013, del total de referencias bibliográficas contenidas en los sílabos de las asignaturas de tres de las principales carreras de psicología en universidades nacionales de gestión pública, solo un 1,9\% de los mismos pertenecía a obras de autores regionales, siendo los tres autores más citados en los sílabos eran Maritza Montero, Ignacio Martín-Baró, Esther Wiesenfeld, Rubén Ardila y Reynaldo Alarcón (Moya, Visca \& Di Doménico, 2013).

Estos datos convergen con estudios previos (Fierro, Ostrovsky \& Di Doménico, en prensa) donde se sugiere que en varios casos, asignaturas históricas abordan mayoritaria o exclusivamente temáticas o contenidos predominantemente localistas. La identificación de la historia de la psicología con la historia de la psicología argentina, si bien esencial para la formación, acota la visión de la historia de la psicología latinoamericana y transregional. Si bien el valor de una historia de la psicología argentina para el psicólogo argentino es innegable, el tamizar la historia de la psicología internacional a partir de la perspectiva nacional probablemente colabore con reforzar y realimentar el ya crónico aislamiento internacional propio del psicólogo rioplatense, toda vez que existen riesgos reales de provincialismo en la historiografía regional (Caparrós, 1993). A la vez, por las particularidades de la psicología local, no puede garantizarse que a partir de dicho tamiz, las peculiaridades que la Argentina no comparte con otras naciones del territorio no se diluyan o sean ocluidas en los cursos de historia. 


\section{Tabla 5}

Nacionalidad de autores con frecuencia de citación mayor a uno en los programas de historia de la psicología

\begin{tabular}{lccc}
\hline Nacionalidad & Frecuencia & Porcentaje & $\begin{array}{c}\text { Porcentaje } \\
\text { acumulado }\end{array}$ \\
\hline Argentino & 267 & 33.5 & 33.5 \\
Norteamericano & 151 & 18.9 & 52.4 \\
Francés & 131 & 16.4 & 68.9 \\
Alemán & 70 & 8.8 & 77.7 \\
Británico & 44 & 5.5 & 83.2 \\
Espańol & 38 & 4.8 & 88.0 \\
Austríaco & 20 & 2.5 & 90.5 \\
Suizo & 16 & 2.0 & 92.5 \\
Colombiano & 6 & .8 & 93.2 \\
Ruso & 6 & .8 & 94.0 \\
Canadiense & 5 & .6 & 94.6 \\
Chileno & 4 & .5 & 95.1 \\
Chileno & 4 & .5 & 95.1 \\
Brasilero & 3 & .4 & 95.5 \\
Cubano & 3 & .4 & 95.9 \\
Estonio & 3 & .4 & 96.2 \\
Neerlandés & 3 & .4 & 96.6 \\
Varios & 3 & .4 & 97.0 \\
Italiano & 2 & .3 & 97.2 \\
Mexicano & 2 & .3 & 97.5 \\
Venezolano & 2 & .3 & 97.7 \\
\hline & & & \\
\hline
\end{tabular}

La escasez de autores y obras sobre psicología latinoamericana forma un vacío que potencilamente podría utilizarse para capitalizar desde una perspectiva comparativa y de conjunto la psicología rioplatense y las diversas psicologías 'nativas' de países vecinos, desnaturalizando y poniendo en relieve de esta forma las características de la primera, a menudo en disonancia con los rasgos de la psicología en países como Perú, México y Colombia (Ardila, 1979; Alarcón, 2002a). Por caso, dos problemas-objeto de asignaturas históricas que son especialmente 
interesantes para la formación académica del psicólogo argentino son, en primer lugar, las diversas reacciones antipositivistas o "espiritualistas" (Alarcón, 2002a, p. 25) de primeras décadas del siglo XX que en diversos países de la región se inspiraron en el vitalismo, el idealismo, la fenomenología y el existencialismo para criticar las aproximaciones científicas, experimentales y cuantitativas de la psicología y proponer en su lugar perspectivas y aproximaciones filosóficas, metafísicas, intuicionistas y axiológicas a la psicología (Alarcón, 2004; Carpintero, 2005).

En segundo lugar, el fenómeno de la casi total extinción de la psicología experimental y comparada en Argentina posterior a dicha reacción positivista, a la institucionalización del psicoanálisis y a la creación de los grados en la década de 1950. Un análisis de tales cuestiones, necesariamente abordadas por autores latinoamericanos, permite contextualizar y contrastar críticamente a la Argentina en la región en tanto caso peculiar del conjunto toda vez que se sostiene que "la psicología latinoamericana actual es predominante empírica, objetiva y cuantitativa” (Ardila, 2004, p. 318). En tal sentido, es necesaria una perspectiva de conjunto de la historia regional para caracterizar a la psicología argentina: una psicología que, según un epistemólogo de la disciplina, aún hacia la década de 1990 registraba una casi nula investigación científica en comparación con la producción de ensayos y conjeturas sin refutaciones, lo que se institucionalizaba en dos 'modelos' de universidad:

Uno, el modelo de universidad profesionalista, reaccionaria y burocrática que, disociada del sistema productivo, extiende títulos al por mayor y convierte a sus egresados en cómplices del subdesarrollo endémico que padece el país; el otro, el modelo de universidad que agrega al proceso de enseńanza-aprendizaje el ingrediente dinámico para que podamos acostarnos con el saber, es decir, la universidad científica, progresista y crítica que, asociada al sistema productivo, estimula la investigación original entre sus docentes y sus alumnos, y mantiene actualizados a sus egresados para que puedan adecuar sus acciones expertas al cambio y a la evolución. En suma, y entre otras cosas, una universidad auténtica se distingue de una mera escuela porque pro- 
mueve la investigación, porque enseña a producir conocimiento y no solo a reproducirlo. (Serroni-Copello, 1990, p. 17)

A su vez, es factible que la consideración de la psicología internacional en un segundo plano signifique no capitalizar una oportunidad vital para exponer al alumno a una perspectiva pluralista, abarcativa y heterogénea de la disciplina que le permita superar los reduccionismos académicos regionales, toda vez que, pasadas dos décadas del siglo XXI, estos difícilmente puedan seguir pasando como 'originalidad vernácula' o valiente resistencia hacia discursos exógenos. Este pluralismo, deseable en toda asignatura, es esencial en las asignaturas históricas, especialmente en las que — como sucede en Argentina- constituyen cursos introductorios del ciclo básico. Finalmente, este pluralismo teórico, conceptual y metodológico es parte esencial de la 'perspectiva' que otorga la educación histórica (Smith, 2007, 2016; Vezzetti, 2007). Como posible causa del fenómeno relevado, puede conjeturarse que existen criterios para la selección de materiales bibliográficos, tal como son ejecutados por los responsables de las asignaturas al diseñar los sílabos, que no consideran la nacionalidad de los historiadores como un parámetro al momento de confeccionar las propuestas docentes. En tal sentido, la Figura 1 y la Figura 2 permitirían suponer que al momento de diseñar los sílabos de asignaturas históricas, la familiaridad de los titulares con el material bibliográfico y la autoría de dichos materiales por los propios responsables de las asignaturas obran en los hechos como un parámetro más relevante que el de la representatividad de autores latinoamericanos o de historiografía iberoamericana. 


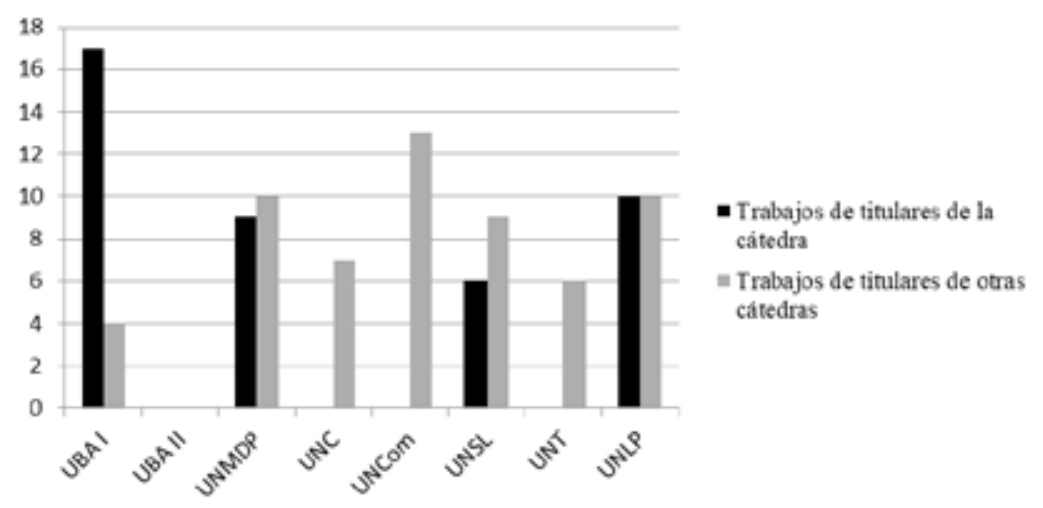

Figura 1. Contenidos bibliográficos de autores argentinos titulares de cátedra en función de cursos y titularidad histórica de las asignaturas

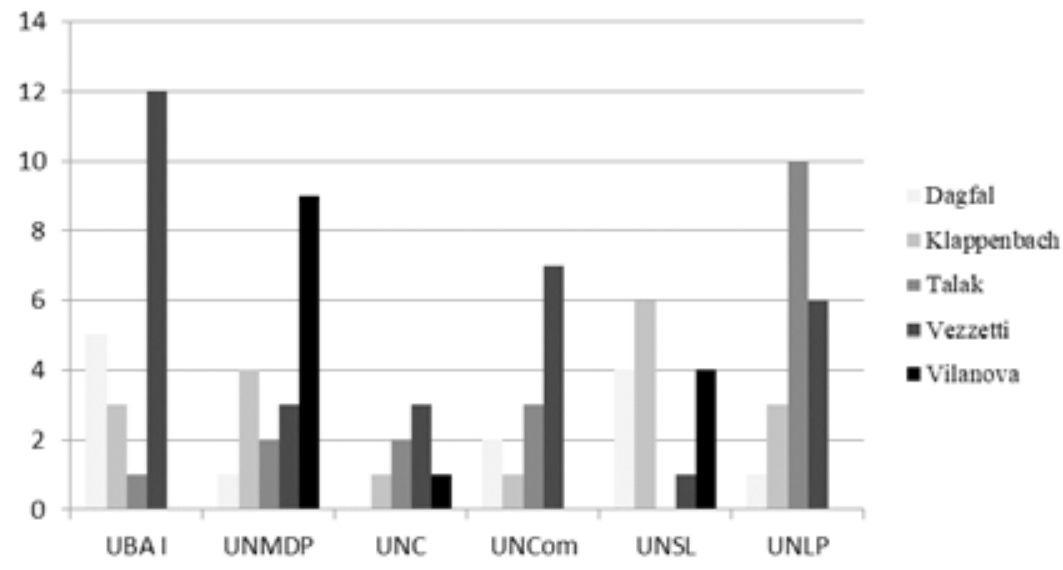

Figura 2. Frecuencia de citación de trabajos de autores argentinos titulares de cátedra en función de asignaturas y titularidades 


\section{Ausencia parcial y total de escuelas, corrientes y orientaciones psicológicas específicas}

Si consideramos como parámetro consideraciones de conjunto sobre la historia de la psicología (Benjamin, 2008; Madsen, 1988; Richards, 1987), existe cierto canon estable aunque siempre revisable y perfectible de autores relevantes para el desarrollo de la disciplina. De acuerdo a los datos relevados y a estudios previos (Fierro \& Di Doménico, 2015), existe cierta disonancia entre tal canon internacional y la enseńanza de la historia de la psicología en Argentina, al menos en algunas asignaturas. Psicólogos históricos significativos como Gordon Allport, James Angell y Jerome Bruner, junto con filósofos directamente relevantes para la psicología como Descartes, Dilthey y Morgan (y junto con psicólogos nacionales como Plácido Horas), son referidos solo dos veces en el total del conjunto de las asignaturas (lo que no implica necesariamente que sean comunes a dos sílabos, puesto que en ocasiones los autores se reiteran en el mismo programa). Con mayores implicancias, autores históricamente relevantes para la psicología internacional como Alfred Adler, James Baldwin, Vladimir Bechterev, Franz Brentano, John Dewey, George Dumas, Paul Fraisse, Franz Galton, Carl Jung, Kurt Lewin, Abraham Maslow y Frederic Skinner (y en el ámbito latinoamericano, autores regionales como Ardila y autores argentinos como Alberini, Piñero y Cortada de Kohan) son referidos solo una vez.

Esto implica que escuelas o corrientes históricas internacionales como el psicoanálisis individual, el funcionalismo de Chicago, la reflexología rusa, la temprana psicología del desarrollo norteamericana, la psicología intencionalista, la psicología analítica, la psicología dinámica de grupo, el humanismo y el conductismo operante, junto con tradiciones argentinas como la primera psicología científica y experimental y la psicología filosófica posterior a 1930, solo están representadas desde fuentes primarias y desde los autores arriba referidos en una sola asignatura.

Sin siquiera abrevar en consideraciones acerca de la naturaleza, metodología o alcance que se le impute a la historia y la historiografía 
de la psicología, la completa omisión de escuelas de pensamiento psicológico internacionales - especialmente aquellas 'fundacionales' - es esencialmente distorsiva respecto al complejo acervo teórico de la disciplina, y es factible que tales omisiones conduzcan al alumno a interiorizar una imagen ficticia y parcializada de la historia de la disciplina. Este recorte al pluralismo y a la perspectiva internacional es aún más perjudicial considerando la monoteoría y el aislamiento disciplinar que caracterizó (y aún caracteriza) al grueso de los psicólogos argentinos. Debe recordarse que de acuerdo a variados reportes (Merced et al., 2017; Richards, 2005; Samper et al., 2008), a nivel internacional es estándar la inclusión de contenidos sobre conductismo, funcionalismo, psicoanálisis, Gestalt, psicofísica, psicología experimental, estructuralismo, psicología cognitiva, psicometría y humanismo en cursos de historia.

Un apartado específico merecería el hecho de que el humanismo, o la 'tercera fuerza', se presente desde fuentes primarias en solo una de las cátedras relevadas. Los humanistas, y entre ellos el equipo de Carl Rogers especialmente, fueron pioneros tanto en la formulación de múltiples innovaciones teóricas, como en la formulación, a nivel tecnológico, de psicoterapias basadas en investigaciones empíricas contrastadas y que, por tanto, minimizaban la especulación de consultorio o la autovalidación circular de la teoría clínica a partir de casos aislados, todo esto aún durante la vida del propio Freud. La exclusión de la tercera fuerza de los contenidos y núcleos temáticos de asignaturas históricas, a la vez que reduce la amplitud teórica de opciones expuestas al alumno (vulnerando el pluralismo usualmente perseguido por los cursos históricos introductorios), coarta el acceso de aquel a la comprensión de, por caso, el hecho de que 'clínica' no es sinónimo de 'psicoanálisis', y del hecho de que existieron diversas alternativas psicoclínicas entre finales del siglo XIX y la segunda guerra mundial.

En otras palabras, oculta el hecho histórico de que hacia 1940, el psicoanálisis era una alternativa clínica entre otras: en un sentido histórico hallándose a pie de igualdad respecto de estas últimas, y en un sentido científico empezando a ser superado por psicoterapias contrastadas por ensayos y tests empíricos sistemáticos. La incorporación de 
estas temáticas en cursos introductorios y a la luz de la historia de la disciplina fomentan la revisión y crítica de asertos sobre la faz profesional de la disciplina (Vande Kemp, 2002), en este caso axiomas psicoclínicos que los psicólogos argentinos han concebido como dogmas durante décadas y que pueden concebirse como problemas teóricos, filosóficos y metodológicos de la denominada 'scientist-practitioner gap'(Cautin, 2011).

A la luz de recientes discusiones historiográficas acerca de la historia policéntrica de la psicología (Brock, 2014) y de discusiones curriculares acerca de la necesidad de internacionalizar los cursos de historia (Pickren, 2012), se torna evidente que una asignatura histórica de carácter crítico, si bien no es susceptible de abarcar con profundidad la totalidad de las corrientes y escuelas psicológicas, sí debería exponer deliberadamente una narrativa multiforme y heterogénea. En este sentido, no sería esperable en una asignatura histórica la exclusión que representa la completa omisión de contenidos sobre escuelas psicológicas enteras (como el humanismo, el conductismo y la Gestalt); escotomización presente en algunas de las asignaturas relevadas.

\section{Predominio de fuentes secundarias y sus implicancias}

El predominio de fuentes secundarias corroboraría lo arriba expuesto respecto a la prevalencia de la historia de la psicología argentina, considerando que la mayoría de las referencias de autores locales son contemporáneas y necesariamente no son fuentes primarias. Si bien las fuentes secundarias son un requisito ineludible en la enseńanza de la historia (para evidenciar los sesgos historiográficos, para ejemplificar el revisionismo histórico, para mostrar la producción actual y constante acerca de la historia de la disciplina y para maximizar la accesibilidad de ciertos contenidos al alumno, entre otras), debe notarse que las fuentes primarias en la enseñanza de la historia de la ciencia son igual o más importantes. Los psicólogos-historiadores acuerdan que aquellas, nucleares a la historiografía crítica, deberían agregarse a estas, y no a la inversa (Vaughn-Blount et al., 2009).

A la luz del énfasis de la historia crítica en las fuentes primarias (cuya omisión dio origen a reconstrucciones históricas presentistas y 
whiggistas, y cuya revalorización se debió a los historiadores que revisaron tales reconstrucciones), enseñar, aprender o investigar en historia requiere un fundamentado trabajo de lectura y análisis de fuentes primarias auxiliado por fuentes secundarias, comentarios y análisis de historiadores (Benjamin, 2009). Reposar mayoritariamente en fuentes secundarias (o terciarias, tales como las fichas de cátedra o los manuales de historia) implica maximizar el riesgo de que ciertas reconstrucciones históricas interesadas sean cedidas acríticamente al alumno, puesto que este probablemente se remita a estas fuentes frente a la complejidad de ciertas temáticas expuestas en fuentes primarias.

El recurso a la lectura de las obras históricas desde una perspectiva historicista (es decir, el diálogo entre el alumno y el pasado disciplinar, en lo posible sin mediaciones distorsivas) es el núcleo crítico de la historiografía revisionista (Barnes \& Greer, 2016; Woodward, 1980). Adicionalmente, el análisis de las fuentes primarias y su cotejo con las reconstrucciones secundarias es precisamente la actividad que ciertos autores ubican como aquello que estimula el pensamiento crítico en espacios curriculares metateóricos (Carroll, 2013; Henderson, 1995; Zehr, 2000a; 2000b). A la luz de la adopción de un enfoque historiográfico 'crítico' por todas las asignaturas, si se considera el énfasis de la historia crítica de la psicología en las fuentes primarias y en la producción de revisiones históricas actualizadas, futuras indagaciones deberán indagar el sentido estricto atribuido por cada asignatura a la criticidad en la historia.

Respecto de la longevidad de las fuentes secundarias (y sin detenernos en el hecho de que es poco factible que un libro completo referenciado en un programa sea de hecho enseñado como contenido), debemos remarcar que en el campo científico a menudo no son los libros sino los papers los que vehiculizan el conocimiento novedoso, especialmente en comparación con capítulos de libros de único autor cuando tales libros o autores no sean obras clásicas, es decir fuentes primarias (Carpintero, 2010). Esto aplica a Historia de la Psicología como especialidad y como subdisciplina con una producción constante de conocimiento sistemático novedoso. 


\section{Conclusiones}

A partir de relevar y analizar la 'duda crónica' sobre el valor de la historia de la psicología, se concluye que dicha historia es, a pesar de los cuestionamientos, un área central para la formación del psicólogo. Esta imagen se refuerza para el caso de la psicología universitaria argentina consideradas sus peculiaridades y déficits, que se entroncan en factores compartidos y factores idiosincrásicos propios de la psicología académica latinoamericana.

La enseñanza de una historia crítica, pluralista, historicista y no-partidaria iría directamente en contra tanto de la formación reductivamente monoteórica y dogmática, como del clinicismo y del practicismo insignes de la psicología argentina que, de acuerdo a múltiples relevamientos, constituyen debilidades endémicas del territorio y de la región. Esto, sin embargo, requiere de revisiones curriculares sistemáticas donde los objetivos curriculares de los cursos históricos y su contribución al perfil de graduado, especialmente en lo referido a la estimulación de un talante crítico, reflexivo y orientado por la investigación, se solapen y articulen con los objetivos de otras asignaturas y del curriculum en su totalidad. En línea con esto, si bien los contenidos y enfoques de cualquier asignatura varían concomitantemente con los objetivos prefijados que ella propone, es necesario y provechoso que tales objetivos se articulen directamente con los objetivos de los planes de estudio, y con las prescripciones contenidas en los estándares de formación ministeriales y en las pautas curriculares -establecidas consensuadamente por los propios profesionales- para la formación integral del psicólogo rioplatense en tanto ciudadano de la república de la ciencia regional e internacional.

A partir del análisis del estado actual de la enseñanza de la historia en universidades argentinas, se concluye que si bien la adopción de enfoques 'críticos' por parte de las asignaturas viabiliza la concreción de las múltiples funciones de la historia, la total omisión de ciertas escuelas o de sus psicólogos representativos, o la prevalencia de la historia de la psicología local por sobre la regional y la internacional en ciertas 
asignaturas dificultaría tal concreción. Esto es especialmente relevante para la cuestión del pluralismo: solo una conceptualización que muestre la naturaleza heterogénea, diversa y multiforme de la psicología y su historia permite demostrar lo problemático de intentar reducir la psicología contemporánea a un enfoque o sistema específico (tanto más si se pretende reducir la disciplina a un enfoque psicológico centenario).

A partir de un uso racional y justificado de los diversos enfoques teóricos y herramientas pedagógicas disponibles para la educación histórica de los psicólogos, y atendiendo e interpelando de forma directa los ya longevos sesgos de la formación universitaria en psicología en Argentina, consideramos que la historiografía como contenido curricular es un insumo central para permitir que la psicología rioplatense en su conjunto pueda optar por uno de los dos caminos que, de acuerdo a Klappenbach (2015), constituyen la 'encrucijada' ante la cual se halla la disciplina en el país del Cono Sur. Se considera necesario profundizar el debate al interior de las asignaturas en torno a la instrumentalización concreta del sentido de la historia de la psicología en la formación universitaria, y en torno a los modelos historiográficos y epistemológicos que dan unidad y coherencia a los enfoques que caracterizan a las asignaturas. Se proyecta que a través de la sistematización de la enseñanza de la historia de la psicología con las características arriba definidas, que de ninguna manera vulneran la libertad de cátedra ni constituyen una normativa monolítica unívoca, tales debates redundarán en un mejoramiento fundamental en la formación de los psicólogos rioplatenses en tanto integrantes de la psicología latinoamericana.

\section{Referencias}

Abd-El-Khalick, F. \& Lederman, N. (2000). Influence of History of Science Courses on Students' Views of Nature of Science. Journal of Research in Science Teaching, 37, 1057-1095. https:// doi.org/10.1002/1098-2736(200012)37:10<1057::AIDTEA3>3.0.CO;2-C 
Alarcón, R. (2002a). Orientaciones teóricas de la Psicología en América Latina. En R. Alarcón (Comp.), Estudios sobre Psicología Latinoamericana (pp. 11-66). Lima: Editorial Universitaria Ricardo Palma.

Alarcón, R. (2002b). ¿Hacia dónde va la psicología latinoamericana?. En R. Alarcón (Comp.), Estudios sobre Psicología Latinoamericana (pp. 211-219). Lima: Editorial Universitaria Ricardo Palma.

Alarcón, R. (2002c). La investigación psicológica en América Latina: Un enfoque histórico. En R. Alarcón (Comp.), Estudios sobre Psicología Latinoamericana (pp. 67-138). Lima: Editorial Universitaria Ricardo Palma.

Alarcón, R. (2004). Medio Siglo de Psicología Latinoamericana: Una Visión de Conjunto. Revista Interamericana de Psicología, 38, 307-316.

Alonso, M. (1999). Psicología en Argentina. En M. Alonso \& A. Eagly (Eds.), Psicología en las Américas (pp. 25-45). México, D.F.: Sociedad Interamericana de Psicología.

Alonso, M. \& Klinar, D. (Noviembre, 2016). Los Psicólogos en Argentina. Relevamiento cuantitativo 2015. Trabajo presentado en el VIII Congreso Internacional de Investigación y Práctica Profesional en Psicología, Buenos Aires, Argentina.

Anónimo. (1987). Encuesta: Opinión de los estudiantes [sobre la formación del psicólogo]. Actualidad Psicológica, 12, 14.

Ardila, R. (1969). Desarrollo de la psicología latinoamericana. Revista Latinoamericana de Psicología, 1, 63-71.

Ardila, R. (1979). La Psicología en Argentina: Pasado, presente y futuro. Revista Latinoamericana de Psicología, 11, 71-91.

Ardila, R. (1984). Factores socioculturales en el desarrollo de la psicología: el caso de América Latina. Revista de Historia de la Psicología, 5, 41-49.

Ardila, R. (1986). La psicología en América Latina. Pasado, presente y futuro. México, D.F.: Siglo XXI. 
Ardila, R. (2004). A Psicologia Latinoamericana: El Primer Medio Siglo. Revista Interamericana de Psicología, 38, 317-322.

Arias, W. (En prensa). Enseñanza de la Historia de la Psicología en la Universidad Católica de San Pablo. En T. Caycho (Ed.), Estudios sobre historia de la psicología en el Perú y Latinoamérica. Lima: UIGV Arias, W. (2014). Historia y actualidad de la psicología peruana. En G. Salas (Ed.), Historias de la Psicología en América del Sur (pp. 163195). La Serena, Chile: Nueva Mirada.

Arias, W. \& Oblitas, A. (2014). Aprendizaje por descubrimiento vs. Aprendizaje significativo: Un experimento en el curso de historia de la psicología. Boletim Academia Paulista de Psicologia, $34,455-471$

Baker, D. (2002). Historical Understanding and Teaching in Professional Psychology. History of Psychology, 5, 219-223. https:// dx.doi.org/10.1037/1093-4510.5.3.219

Barnes, M. \& Greer, S. (2016). The history specialist in psychology: From avocation to professionalization. History of Psychology, 19, 192-208. https://dx.doi.org/10.1037/hop0000027.

Bartos, S. \& Banks, A. (2015). How do students learn critical thinking? Challenging the osmosis model. History y Philosophy of Psychology, 16, 36-40.

Beins, B. (2016). A History of the History of American Psychology: Where It Has Been and Where It Is Now. En W. D. Woody, R. Miller y W. Wozniak (Eds.), Psychological Specialties in Historical Context. Enriching the Classroom Experience for Teachers and Students (pp. 18-32). Society for the Teaching of Psychology. Recuperado de http://teachpsych.org/ebooks/.

Benjamin, L. (2005). A history of clinical psychology as a profession in America (and a glimpse at its future). Annual Review of Clinical Psychology, 1, 1-30. https://doi.org/10.1146/annurev. clinpsy.1.102803.143758

Benjamin, L. (2008). History of Psychology: Original Sources and Contemporary Research (3ra Ed.). Nueva York, NY: Wiley-Blackwell Publishers. 
Benjamin, L. (2010). History of Psychology as a Capstone Course. En D. Dunn, B. Beins, M. McCarthy y W. G. Hill (Eds.), Best Practices for Teaching Beginnings and Endings in the Psychology Major (pp. 171-186). Oxford, Inglaterra: Oxford University Press.

Benjamin, L. \& Baker, D. (2009). Recapturing a Context for Psychology. The Role of History. Perspectives on Psychological Science, 4, 97-98. https://doi.org/10.1111/j.1745-6924.2009.01097.x

Bhatt, G. \& Tonks, R. (2002). What lies in the future of teaching the history of psychology? History and Philosophy of Psychology Bulletin, 14, 2-9.

Biglieri, F., Brisuela Blume, L., Fierro, C. \& Bruna, O. (octubre, 2016). Evaluación del impacto de revisiones curriculares en el contexto de los procesos de acreditación: Un estudio socio-bibliométrico de asignaturas psicoclinicas de UBA, UNLP y UNMDP. Trabajo presentado en el II Congreso Latinoamericano para el Avance de la Ciencia Psicológica, Buenos Aires, Argentina.

Brock, A. (2014). What is a polycentric history of psychology? Estudos e Pesquisas em Psicología, 14, 646-659. https://doi.org/10.12957/ epp.2014.12565

Brock, A. (2016a). The future of the history of psychology revisited. History of Psychology, 19, 175-191. http://dx.doi.org/10.1037/ hop0000037

Brock, A. (2016b). The History of Psychology in Germany . History of Psychology, 19, 73-375. https://doi.org/10.1037/h0101589

Brock, A. \& Harvey, M. (2015). The status of the history of psychology course in British and Irish psychology departments. European Yearbook of the History of Psychology, 1, 13-36. https:// doi.org/10.1484/J.EYHP.5.108400

Brush, S. (1974). Should the History of Science Be Rated X? Science, 183, 1164-1172. https://doi.org/10.1126/science.183.4130.1164

Buchanan, T. (2002). Historically grounding the practice of psychology: Implications for professional training. History of Psychology, 5, 240-248. https://doi.org/10.1037/1093-4510.5.3.240 
Caparrós, A. (1993). Historias regionales desde la psicología: Notas historiográficas. Revista de historia de la psicología, 14, 9-92.

Capshew, J. (2014). History of Psychology since 1945. A North American Review. En R. Backhouse \& P. Fontaine (Eds.), A Historiography of the Modern Social Sciences (pp. 144-182). Nueva York: Cambridge University Press.

Carpintero, H. (2005). La psicología iberoamericana. Una perspectiva según el método histórico de las generaciones. Revista de Historia de la Psicologia, 26, 41-56.

Carpintero, H. (2007). La historia de la psicología iberoamericana. Contribuciones a su estudio en la 'Revista de Historia de la Psicología'. Revista de Historia de la Psicología, 28, 25-33.

Carpintero, H. (2010). Los géneros literarios en Psicología. Revista de Historia de la Psicología, 31, 9-30.

Carpintero, H. \& Peiró, J. M. (Dirs.). (1981). Psicología contemporánea: Teoría y métodos cuantitativos para el estudio de su literatura cientifica. Valencia: Alfaplus.

Carpintero, H. \& Tortosa, F. (1990). Aplicaciones de la metodología bibliométrica a la historia de la psicología: Una visión de conjunto. En F. Tortosa, L. Mayor y H. Carpintero (Eds.), La Psicologia Contemporánea desde la Historiografia (pp. 275-314). Barcelona, España: PPU.

Carroll, D. (2013). Counterfactual Thinking in the History of Psychology Course. Psychology Learning \& Teaching, 12, 76-82. https://doi.org/10.2304/plat.2013.12.1.76

Carroll, D. W. (2006). Thinking about historical issues: Debates in the history and systems class. Teaching of Psychology, 33, 137-140.

Carroll, D., Keniston, A. \& Peden, B. (2008). Integrating Critical Thinking with Course Content. En D. Dunn, J. Halonen y R. Smith (Eds.), Teaching Critical Thinking in Psychology: A Handbook of Best Practices (pp. 101-115). Londres: Blackwell. https:// doi.org/10.1002/9781444305173.ch9 
Cautin, R. (2011). Invoking History to Teach about the ScientistPractitioner Gap. History of Psychology, 14, 197-203. https://doi. org/10.1037/a0022897

Chisvert-Perales, M., Monteagudo-Soto, M. J. \& Mestre, V. (2016). The history of psychology course in Spanish psychology curricula: Past, present, future. History of Psychology, 19, 154-168. https://dx.doi.org/10.1037/hop0000024.

Christie, J. (2005). El Desarrollo de la Historiografía de la Ciencia. En S. Martínez y G. Guillaumin (Comps.), Historia, Filosofía y Enseñanza de la Ciencia (pp. 43-66). México, D.F.: Universidad Autónoma de México.

Coll, C. (1996). Psicología y Currículum. Buenos Aires: Paidós.

Collins, A. \& Bunn, G. (2016). The Shackles of Practice: History of Psychology, Research Assessment, and the Curriculum. En S. Klempe y R. Smith (Eds.), Centrality of History for Theory Construction in Psychology (pp. 91-109). Cham: Springer. doi: 10.1007/978-3-319-42760-7_5.

Courel, R. \& Talak, A. M. (2001). La formación académica y profesional del psicólogo en Argentina. En J. Toro y J. Villegas (Eds.), Problemas centrales para la formación académica y el entrenamiento profesional del psicólogo en las Américas, Vol. 1 (pp. 21-83). Buenos Aires: JVE.

Dagenbach, D. (1999). Some Thoughts on Teaching a Pluralistic History in the History and Systems of Psychology Course. Teaching of Psychology, 26, 22-28. https://dx.doi.org/10.1207/ s15328023top2601_4

Dagfal, A. (2014). La Identidad Profesional como Problema: El caso del "Psicólogo-Psicoanalista" en la Argentina (1959-1966). Psicología em Pesquisa, 8, 97-114. https://doi.org/10.5327/ Z1982-1247201400010010

Dagfal, A. (noviembre, 2015). El psicoanálisis como una psicología. ¿Herejía o realidad histórica y social? En A. Viguera (Coord.), Debates y Temas Actuales en historia del Psicoanálisis. Simposio 
realizado en el V Congreso Internacional de Investigación en Psicología, La Plata, Argentina.

Danziger, K. (1984). Towards a Conceptual Framework for a Critical History of Psychology. Revista de Historia de la Psicología, 5, 99-107.

Danziger, K. (1994). Does the history of psychology have a future? Theory and Psychology, 8, 467-484. https://doi.org/ 10.1177/0959354394044001

Danziger, K. (1997). The Future of Psychology's History is Not its Past: A Reply to Rappard. Theory \& Psychology, 7, 107-111. https:// doi.org/10.1177/0959354397071009

Danziger, K. (2001). The Historiography of Psychological Objects. En K. Danziger, Problematic Encounter: Talks on Psychology and History (pp. 1-8). Recuperado de http://www.kurtdanziger.com/ Paper\%207.pdf.

Danziger, K. (2013). Psychology and its History. Theory \& Psychology, 23, 829-839. https://doi.org/10.1177/0959354313502746

Deconchy, J. P. (1984). Rationality and Social Control in Orthodox Systems. En H. Tajfel, C. Fraser y J. Jaspars (Eds.), The Social Dimension: Volume I (pp. 425-445). Cambridge: Cambridge University Press. https://doi.org/10.1017/ CBO9780511759154.004

Dehue, T. (1998). Community Historians and the Dilemma of Rigor vs Relevance: A Comment on Danziger and Van Rappard. Theory o Psychology, 8, 653-661. https://doi. org/10.1177/0959354398085004

Dembo, M. (1993). Estrategias y contenidos en la formación del psicólogo. Trabajo presentado en el XXIV Congreso Interamericano de Psicología, Santiago de Chile, Chile.

Di Doménico, C. (1996). Psicología y Mercosur: acerca de la armonizacion curricular. Acta Psiquiátrica y Psicológica de América Latina, 42, 230-242. 
Di Doménico, C. (1999). Psicología y Mercosur: revisión comparativa de los acuerdos sobre formación de psicólogos. Acta Psiquiátrica y Psicológica de América Latina, 45, 24-33.

Di Doménico, C. (2015). Formación de Psicólogos. Aristas de la calidad. Psiencia, 7, 124-132. https://dx.doi.org/10.5872/ psiencia.v7i1.157

Di Doménico, C. \& Piacente, T. (2011). Acreditación en Psicología en el Cono Sur de América. Psicolatina, 22, 1-18.

Di Doménico, C. \& Vilanova, A. (junio, 1999). Estudio comparativo de diseños curriculares en paises del Mercosur. Informe. Trabajo presentado en el XXVII Congreso Interamericano de Psicología, Caracas, Venezuela.

Di Doménico, C. \& Vilanova, A. (septiembre, 2000). Formación básica en psicología en carreras de universidades públicas de Argentina. Trabajo presentado en el I Congreso Hispano Portugués de Psicología, Santiago de Compostela, España.

Ennis, R. (1991). Critical Thinking: A Streamlined Conception. Teaching Philosophy, 14, 5-24. https://doi.org/10.5840/ teachphil19911412

Fernández, M., Aguinaga, M., Gallardo, J. \& Grassi, M. (2011). Evaluación del impacto de la enseñanza de la perspectiva histórica en los alumnos de primer año de la carrera de psicología de la UNLP. En Actas del III Congreso Internacional de Investigación de la Facultad de Psicología de la Universidad Nacional de La Plata (pp. 21-27). La Plata: Universidad Nacional de La Plata. Recuperado de http://www.memoria.fahce.unlp.edu.ar/trab_ eventos/ev.1424/ev.1424.pdf.

Fierro, C. (2015a). La Historia de la Psicología y su Enseñanza a 40 años de 'Should the History of Science Be Rated X? de Stephen Brush. Estudos e Pesquisas em Psicologia, 15, 310-330. https:// doi.org/10.12957/epp.2015.16076

Fierro, C. (2015b). El Rol de la Enseñanza de la Historia de la Psicología en la Formación del Psicólogo: Relevamiento y Análisis de 
Algunos Argumentos sobre su Importancia Curricular. Perspectivas en Psicología, 12, 18-28.

Fierro, C. (2016a). Enseñanza de la Historia de la Psicología y Formación de Psicólogos: Desarrollos y Debates Actuales en Argentina y el Mundo. Arequipa, Perú: Sociedad Peruana de Historia de la Psicología/ADRUS.

Fierro, C. (2016b). Institucionalización y Profesionalización de la Historia de la Psicología como Especialidad en Estados Unidos: Influencias de la Historia, la Sociología y la Filosofía de la Ciencia. Revista Argentina de Ciencias del Comportamiento, 8, 67-92.

Fierro, C. (En prensa). Integrando historiografía crítica, filosofía de la ciencia y estudios sobre enseñanza y formación universitaria en psicología: Problemas y perspectivas. Quaderns en Psicología.

Fierro, C. \& Di Doménico, C. (julio, 2015). Presencia de Autores Históricos desde Fuente Primaria en la Enseñanza de la Historia de la Psicología en Universidades Públicas Argentinas. Trabajo presentado en el XXXV Congreso Interamericano de Psicología, Lima, Perú.

Fierro, C. \& Di Doménico, C. (2016). Pluralismo crítico: Historia de la Ciencia en debates sobre formación en psicología. Quaderns de Psicología, 18, 27-57. https://doi.org/10.5565/rev/ qpsicologia. 1322

Fierro, C. \& Di Doménico, C. (2017). Enseñanza y formación universitaria en Psicología en Argentina: Caracterización de una taxonomía del pensamiento crítico. Cuadernos de Neuropsicologia, 11, 42-79. http://doi.org/10.7714/CNPS/11.1.202.

Fierro, C., Ostrovsky, A. E. \& Di Doménico, C. (2017). Las asignaturas de historia, sistemas y escuelas de la psicología en la formación de los psicólogos (I): Análisis comparativo del segundo nivel de concreción curricular en departamentos americanos y europeos de psicología. Revista de Historia de la Psicología, 38, 16-32. https://doi.org/10.5093/rhp2017a3

Fierro, C., Ostrovsky, A. E. \& Di Doménico, C. (En prensa). Current state of history of psychology teaching and education in 
Argentina: An empirical bibliometric investigation. Teaching of Psychology, 45.

Fuchs, A. \& Viney, W. (2002). The Course in the History of Psychology: Present status and Future concerns. History of Psychology, 5, 3-15. https://doi.org/10.1037/1093-4510.5.1.3

Furumoto, L. (1989). The new history of psychology. En I. Cohen (Ed.), The G. Stanley Hall Lecture Series (Vol. 9) (pp. 5-34). Washington, D.C: APA. https://doi.org/10.1037/10090-001

Gallegos, M. (2009). Movimiento y participación estudiantil en la psicología latinoamericana: consideraciones históricas y futuras perspectivas. Avances en Psicología Latinoamericana, 27, 30-57.

Gallegos, M. (2016). Historia de la psicología y formación en psicología en América Latina: convergencias temáticas. Enseñanza e Investigación en Psicología, 21, 319-335.

García, J. E. (2003). Problemas centrales en la formación académica y el entrenamiento profesional del psicólogo en el Paraguay. En J. Villegas \& J. P. Toro (Eds.), Problemas centrales en al formación académica y el entrenamiento profesional del Psicólogo en las Américas, Vol. 2 (pp. 205-279). Santiago, Chile: Sociedad Interamericana de Psicología.

García, J. E. (2009b). Breve Historia de la Psicología en Paraguay. Psicolatina, 17. Recuperado de http://www.psicolatina.org/17/ paraguay.html.

García, J. E. (2011a). La Enseñanza de la Historia de la Psicología en las Universidades Paraguayas. Revista del Instituto de Investigaciones Psicológicas, 14, 74-96.

García, J. E. (2011b). Enseñanza de la Historia de la Psicología paraguaya. Pensamiento Psicológico, 9, 103-122.

García, J. E. (2014). La relación entre investigación básica y profesión aplicada en la psicología latinoamericana. Revista Peruana de Psicología y Trabajo Social, 3, 109-124.

García, L. N. (2009). La disciplina que no es: los déficit en la formación del psicólogo argentino. Psiencia, 1, 12-23. 
Giménez, M. C. (2002). Algunas reflexiones sobre la historia de la psicología y la formación de psicólogos. Anuario de psicología, 33, 213-224.

Gonzalez, C., Tornimbeni, S., Corigliani, S., Gentes, G., Ginochio, A. \& Morales, M. (2012). Evaluación de competencias requeridas para investigar. Anuario de Investigaciones de la Facultad de Psicologia, 1, 142-151.

González, E. \& Rodríguez, A. (1999). Enseñanza y profesionalización de la psicología en el Uruguay. En C. Di Doménico \& A. Vilanova (Eds.), Formación de Psicólogos en el Mercosur (pp. 83-94). Mar del Plata: Universidad Nacional de Mar del Plata.

González, M. E. (2015a). Las carreras de Psicología, universidad y democracia en Argentina: Notas para una historia reciente. Procesos Históricos: Revista de Historia y Ciencias Sociales, 26, 111-125.

González, M. E. (2015b). Observaciones en torno a los Debates Curriculares en Psicología y las Políticas Universitarias en Argentina. Psiencia, 7, 35-40. https://doi.org/10.5872/psiencia/7.1.141

González, M. E. (2015c). Complejidad de la psicología: Perspectivas actuales y alcances para la formación universitaria. En V. Marínez-Nuñez (Ed.), Avances y Desafios para la Psicologia (pp. 271-279). San Luis: Nueva Editorial Universitaria.

González, M. E. \& Dagfal, A. (2012). El psicólogo como psicoanalista: Problemas de formación y autorización. Intersecciones Psi, 2, 12-18.

Gooday, G., Lynch, J., Wilson, K. \& Barsky, C. (2008). Does Science Education Need the History of Science? Isis, 99, 322-330. https://doi.org/10.1086/588690

Goodwin, J. (1997). The vital role of Psychology's history in Introductory Courses: An Interview with Ludy T. Benjamin, Jr. Teaching of Psychology, 24, 218-221. https://doi.org/10.1207/ s15328023top2403_20 
Harris, B. (1980). Ceremonial Versus Critical History of Psychology. American Psychologist, 35, 218-219. http://dx.doi. org/10.1037/0003-066X.35.2.218

Harris, B. (2011). Letting Go of Little Albert: Disciplinary Memory, History and the Uses of Myth. Journal of the History of the Behavioral Sciences, 47, 1-17. https://doi.org/10.1002/jhbs.20470

Hart, J. \& English, B. (1983). Theoretical orientation change in students following a course in history and systems of psychology. Teaching of Psychology, 10, 37-41. https://doi.org/10.1207/ s15328023top1001_10

Hawes, G. (2003). Pensamiento crítico en la formación universitaria. Talca: Instituto de Investigación y Desarrollo Educacional de la Universidad de Talca.

Henderson, B. (1995). Critical-Thinking Exercises for the History of Psychology Course. Teaching of Psychology, 22, 60-63. https:// doi.org/10.1207/s15328023top2201_18

Hereford, C. (1966). Current Status of Psychology in Latin America. Latin American Research Review, 1, 97-108.

Hilgard, E., Leary, D. \& McGuire, G. (1991). The History of Psychology: A Survey and Critical Assessment. Annual Review of Psychology, 42, 79-107. https://doi.org/10.1146/annurev. ps.42.020191.000455

Jacó-Vilela, A. M. (1999). Formação do psicólogo: Um pouco de história. Interaçóes: Estudos e Pesquisa em Psicologia, 4(8), 79-91.

Jaraba Barrios, B., Guerrero-Castro, J., Gomez Morales, Y. J. \& López López, W. (2011). Bibliometría e historia de las prácticas académicas locales: un esbozo a partir del caso de la psicología en Colombia. Avances en Psicología Latinoamericana, 29, 354-369.

Klappenbach, H. (2000a). El titulo profesional de psicologo en argentina. Antecedentes historicos y situación actual. Revista Latinoamericana de Psicología, 32, 419-446.

Klappenbach, H. (2000b). Historia de la historiografía de la psicología. En J. C. Ríos, R. Ruiz, J. C. Stagnaro y P. Weissman (Comps.), 
Psiquiatría, Psicología y Psicoanálisis: Historia y Memoria (pp. 238-268). Buenos Aires: Polemos.

Klappenbach, H. (2000c). El psicoanálisis en los debates sobre el rol del psicólogo. Argentina, 1960-1975. Revista Universitaria de Psicoanálisis, 2, 191-227.

Klappenbach, H. (2003a). La Globalización y la Enseñanza de la Psicología en la Argentina. Psicología em Estudo, 8, 3-18. https://doi. org/10.1590/S1413-73722003000200002

Klappenbach, H. (2003b). La investigación en carreras de grado de psicología en universidades nacionales de Argentina. En J. Villegas, P. Marasi y J. P. Toro (Eds.), Problemas centrales para la formación académica y el entrenamiento profesional del psicólogo en las Américas (Vol. 3) (pp. 257-271). Universidad Central de Chile: SIP Klappenbach, H. (2004). Psychology in Argentina. En D. Wedding y M. Stevens (Eds.), Handbook of International Psychology (pp. 129-150). New York: Brunner-Routledge.

Klappenbach, H. (2008/2009). Estudio bibliométrico de la Revista de Psicología de la Universidad Nacional de La Plata: 1964-1983 . Revista de Psicología, 10, 13-65.

Klappenbach, H. (2013). Aplicación del enfoque socio-bibliométrico a los estudios en Historia y Formación en Psicología. Trabajo presentado en la XIV reunión nacional y III encuentro nacional de la Asociación Argentina de Ciencias del Comportamiento, Córdoba, Argentina.

Klappenbach, H. (2014). Informes norteamericanos sobre la Psicología en América el Sur anteriores a 1950. En G. Salas (Ed.), Historias de la Psicología en América del Sur (pp. 216-238). La Serena, Chile: Nueva Mirada.

Klappenbach, H. (2015). La formación universitaria en Psicología en Argentina. Perspectivas actuales y desafíos a la luz de la historia. Universitas Psychologica, 14, 937-960. https://dx.doi. org/10.11144/Javeriana.upsy14-3.fupa.

Klappenbach, H. \& Fierro, C. (2016). La investigación histórica de la psicología en la argentina: caracterización y estudio comparativo 
de las temáticas de investigación en los encuentros argentinos de historia de la psiquiatría, la psicología y el psicoanálisis y la revista History of Psychology (2009-2015). Actas del Encuentro Argentino de Historia de la Psiquiatría, la Psicología y el Psicoanálisis, 17, 174-201.

Klappenbach, H. \& Jacó-Vilela, A. M. (2016). The future of the history of psychology in Argentina and Brazil. History of Psychology, 19, 229-247. https://dx.doi.org/10.1037/hop0000030.

Krantz, D. (1972). Schools and systems: The mutual isolation of operant and non-operant psychology as a case study. Journal of the History of the Behavioral Sciences 8, 86-102. https://doi.org/10.1002/1520-6696(197201)8:1<86::AIDJHBS2300080104>3.0.CO;2-B

Kushner, R. (1980). The Prescritive Approach to the Teaching of the History of Psychology Course. Teaching of Psychology, 7, 184185. https://doi.org/10.1207/s15328023top0703_20

Landrum, E. (2016). The History of the Teaching of Psychology: Or, What Was Old is New Again. En W. D. Woody, R. Miller \& W. Wozniak (Eds.), Psychological Specialties in Historical Context. Enriching the Classroom Experience for Teachers and Students (pp. 47-54). Society for the Teaching of Psychology. Recuperado de http://teachpsych.org/ebooks/.

Loredo, J. C. (1998). Relativismo e historiografia de la psicologia. Anuario de Psicologia, 29, 55-62.

Maddux, J. (Octubre, 2016). La psicología social de la percepción y el juicio clinico: La verdad incómoda. Conferencia magistral presentada en el II Congreso Latinoamericano para el Avance de la Ciencia Psicológica, Buenos Aires, Argentina.

Madsen, K. (1988). A History of Psychology in Metascientific Perspective. North Holland: Elsevier.

Manzo, G. (2010). Competencias autopercibidas en estudiantes de psicología del ciclo profesional en la UNMDP. Anuario de Proyectos e Informes de Investigación, 7, 402-413. 
Manzo, G. (2011). Competencias Autopercibidas en Estudiantes de Psicología del Ciclo Profesional en UNMDP. Anuario de Proyectos e Informes de Becarios de Investigación, 8, 402-413.

Manzo, G., Fierro, C. y Di Doménico, C. (octubre, 2016). Formación de posgrado en Psicología: Análisis bibliométrico de la literatura requerida en residencias clinicas de la ciudad de Mar del Plata. Trabajo presentado en el II Congreso Latinoamericano para el Avance de la Ciencia Psicológica, Buenos Aires, Argentina.

Marín, G., Kennedy, S. \& Boyce, B. C. (1987). Latin American Psychology: A Guide to Research and Training. Washington, D.C.: APA.

Mariñelarena-Dondena, L. \& Klappenbach, H. (2016). Enseñanza de la psicología positiva en la Argentina: un estudio socio-bibliométrico. Psiencia. Revista Latinoamericana de Ciencia Psicológica, 8, doi: 10.5872/psiencia/8.2.141.

Martín-Baró, I. (1986). Hacia una psicología de la liberación. Boletín de Psicología, 22, 219-231

Matthews, M. (2015). Science Teaching: The Contribution of History and Philosophy of Science. New York: Routledge.

McPherson, M. (1979). Different approaches to teaching the history of psychology: Excerpts from a 1977 APA symposium. Psychological Record, 29, 65-70. https://doi.org/10.1007/BF03394590

Merced, M., Stutman, Z. \& Mann, S. (2017). Teaching the History of Psychology: A Content Analysis of Course Syllabi from Doctor of Psychology Programs. Psychology Learning \& Teaching. https:// doi.org/10.1177/1475725717729909

Menéndez, P. \& Acosta, S. (2011). Formación teórico-clínica de Psicología en Argentina: Universidades de Buenos Aires, de Córdoba y de Rosario. Anuario de Investigaciones, 18, 61-67.

Mestre, M. (2007). La Historia de la Psicología en el futuro de la Enseńanza Universitaria. Revista de Historia de la Psicología, 28, 77-83.

Miguel, S. \& Dimitri, P. J. (2013). La investigación en bibliometría en la Argentina: Quiénes son y qué producen los autores argentinos 
que realizan estudios bibliométricos. Información, Cultura $y$ Sociedad, 29, 117-138.

Monroy-Nasr, Z., Álvarez-Díaz de León, G. \& León-Sánchez, R. (2009). The Challenge of Teaching History of Psychology: a New Curriculum, a New Program and the Students' Previous Ideas. Trabajo presentado en la Biennial Conference de la International History, Philosophy and Science Teaching Group, Notre-Dame, Francia. Recuperado de http://www3.nd.edu/ ihpst09/papers/ Monroy\%20manuscript.doc.

Montero, I. \& León, O. (2007). A guide for naming research studies in Psychology. International Journal of Clinical and Health Psychology, 7, 847-862.

Morais Nicaretta, M. (2009). A formação do mercado das psicoterapias nos Estados Unidos da América e no Brasil: Psicanálise, Psicologia Clínica e Psicoterapias. Boletim Academia Paulista de Psicologia, 29, 20-36.

Moya, L., Visca, J. \& Di Doménico, C. (2013). Presencia de Autores Latinoamericanos en la Formación Básica de los Psicólogos en Argentina: El Caso de Tres Universidades Públicas. En N. Zícavo Martinez (Comp.), Construyendo una Psicología Comprometida en América Latina (pp. 2-18). Chile: Universidad del Bío Bío.

Norcross, J., Hailstorks, R., Aiken, L. \& Pfund, R. (2016). Undergraduate Study in Psychology: Curriculum and Assessment. American Psychologist, 71, 89-101. http://dx.doi.org/10.1037/ a0040095.

Ostrovsky, A. (2010). Las historias críticas de la psicología desde la perspectiva de género. Pensando sus influencias y posibilidades. Estudos e Pesquisas em Psicologia, 10, 911-929. https://doi. org/10.12957/epp.2010.8933

Ostrovsky, A. \& Di Doménico, C. (2007). Formación de grado en Psicología: Opiniones controversiales de profesores Argentinos, Latinoamericanos y Europeos. En Actas de las XIV Jornadas de Investigación de la Facultad de Psicología de la Universidad de Buenos Aires (pp. 389-392). Buenos Aires: Universidad de 
Buenos Aires. Recuperado de http://www.aacademica.org/000073/320.pdf.

Parra Herrera, Y., Colunga Santos, S. \& Carvajal Hernández, B. (2016).

Niveles y patrones de logro para la formación de la competencia.

Humanidades Médicas, 16, 475-488.

Piacente, T. (1998). Psicoanálisis y formación académica en Psicología. Acta Psiquiátrica y Psicológica de América Latina, 44, 278-284.

Pickren, W. (2012). Internationalizing the History of Psychology Course in the USA. En F. Leong, W. Pickren, M. Leach \& A. Marsella (Eds.), Internationalizing the Psychology Curriculum in the United States (pp. 11-28). Nueva York: Springer.

Polanco, F. \& Calabresi, C. (2011). La enseñanza de la Psicología en la Argentina. Entrevista con Hugo Klappenbach. Universitas Psychologica, 10, 613-626.

Popper, K. (1957/1967). La ciencia: conjeturas y refutaciones. En K. Popper (Comp.), El desarrollo del conocimiento científico. Conjeturas y refutaciones (pp. 43-79). Buenos Aires: Paidós.

Richards, G. (1987). Of What is History of Psychology a History? British Journal of the History of Science, 20, 201-211.

Richards, G. (2005). The pattern of History of Psychology teaching on British undergraduate Psychology courses. Psychological Teaching Review, 11, 12-24.

Robinson, D. (1979). The History of Psychology and the Ends of Instruction. Teaching of Psychology, 6, 4-6. https://doi. org/10.1207/s15328023top0601_2

Roberts, D. (1982). Developing the Concept of "Curriculum Emphases” in Science Education. Science Education, 66, 243260. https://doi.org/10.1002/sce.3730660209

Rojo González, M. D. (1993). Criterios y presupuestos en la formación del psicólogo. Trabajo presentado en el XXIV Congreso Interamericano de Psicología, Santiago de Chile, Chile.

Rosa, A. (2002). La historia en la formación universitaria. Anuario de Psicología, 33, 209-212. 
Rutherford, A. (2002). Back to the classroom... and beyond. A comment on Bhatt and Tonks. Bulletin for the History and Philosophy of Psychology, 14, 17-19.

Rutherford, A. (2013). Teaching Diversity: What Can History Offer? History of Psychology, 16, 1-5.

Rutherford, A. (2014). Historiography. En T. Teo (Ed.), Encyclopedia of Critical Psychology (pp. 866-872). Nueva York: Springer.

Rutherford, A. \& Pickren, W. (2015). Teaching the History of Psychology: Aims, Approaches and Debates. En D. Dunn (Ed.), The Oxford Handbook of Undergraduate Psychology Education (pp. 521-532). Londres, Inglaterra: Oxford University Press. https:// doi.org/10.1093/oxfordhb/9780199933815.013.040

Salas, G. (2014). Pasado y Presente de la Psicología Científica en Chile: Profesionalización, instituciones y divulgación científica. En G. Salas (Ed.), Historias de la Psicología en América del Sur (pp. 95-114). La Serena, Chile: Nueva Mirada.

Samelson, F. (1980). J. B. Watson's Little Albert, Cyril Burt's Twins, and the Need for a Critical Science. American Psychologist, 35, 619-625. http://dx.doi.org/10.1037/0003-066X.35.7.619

Samper, P., Mestre, V., Tur, A. M., Orero, A. \& Falgás, M. (2008). La situación de la Historia de la Psicología en el currículo del Psicólogo. Revista de Historia de la Psicología, 29, 211-221.

Sánchez, F., Rivero, A. \& Pérez, D. (2000). Creando historias de la psicología. Algunos efectos de la enseñanza de la asignatura. Historia de la Psicología, 21, 25-34.

Sanz, R. (1997). Seis problemas para la Historia de la Psicología. Cuadernos Argentinos de Historia de la Psicología, 3, 67- 76.

Saposnik, G., Redelmeier, D., Ruff, C. \& Tobler, P. (2016). Cognitive biases associated with medical decisions: a systematic review. BMC Medical Informatics and Decision Making, 16, http://doi. org/10.1186/s12911-016-0377-1.

Serroni-Copello, R. (1990). Las investigaciones psicológicas y la universidad desexualizada. Boletín Argentino de Psicología, 3, 17-20. 
Serroni-Copello, R. (1997a). Expansión discursiva y recursiva en la evolución de la psicología. En R. Serroni-Copello (Comp.), Diálogo, racionalidad y salud mental. (pp. 27-42). Buenos Aires: ADIP.

Serroni-Copello, R. (1997b). Disonancias estructurales en el desarrollo de la psicología en la Argentina. En R. Serroni-Copello (Comp.), Diálogo, racionalidad y salud mental (pp. 57-72). Buenos Aires: ADIP.

Slapak, S. (1987). La carrera de psicología en la Universidad de Buenos Aires. Actualidad Psicológica, 12, 3.

Smith, A. (1982). Different Approaches for Teaching the History of Psychology Course. Teaching of Psychology, 9, 180-182. https:// doi.org/10.1207/s15328023top0903_12

Smith, R. (2007). Why history matters. Revista de Historia de la Psicologia, 28, 125-146.

Smith, R. (2016). History of Psychology: What For? En S. H. Klempe y R. Smith (Eds.), Centrality of History for Theory Construction in Psychology (pp. 3-28). Cham: Springer. https://doi. org/10.1007/978-3-319-42760-7_1

Sokal, M. (1984a). James McKeen Cattell and American Psychology in the 1920s. En J. Brozek (Ed.), Explorations in the History of Psychology in the United States (pp. 273-323). Lewisburg: Bucknell University Press.

Sokal, M. (1984b). History of Psychology and History of Science: Reflections on two Subdisciplines, Their Relationship and their Convergence. Revista de Historia de la Psicología, 5, 337-347.

Tao, A. \& Li, X. (2014a). Relationship between Critical Thinking and Personality Based on the History of Psychology Teaching. American International Journal of Social Science, 3, 88-93.

Tao, A. \& Li, X. (2014b). Achievement Motivation Improving and its Relationship with Personality Based on the Teaching Reform of Psychology. International Journal of Education and Research, 2, 341-346. 
Toro, J. P. \& Villegas, J. (Eds.). (2001). Problemas Centrales en la Formación Académica y el Entrenamiento Profesional del Psicólogo en las Américas (Vol. I). Buenos Aires: JVE.

Tortosa, F., Mayor, L. \& Carpintero, H. (1990). La Historiografía de la Psicología: Orientaciones y Problemas. En F. Tortosa, L. Mayor y H. Carpintero (Eds.), La Psicología Contemporánea desde la Historiografía (pp. 25-48). Barcelona: PPU.

Tosi, L. \& Tosi, J. (2014). Escolasticismo: ¿Problema fundamental de la psicología de hoy? Psicolatina, 26, 63-86.

Vainer, A. (1997). Efectos dogmáticos de Lacan en Argentina. Topía, 21, 29-31.

Valdés-Fauly, M. C. \& de la Torre Molina, C. (1985). La historia de la psicologia en sus manuales de estudio. Revista Cubana de Psicologia, 3, 41-49.

van Hoorn, W. (1984). The minimal meaningful context. Revista de Historia de la Psicología, 5, 163-171.

van Strien, P. (1990). Recontextualization as a Contribution of History to Theoretical Psychology. En W. Baker, M. Hyland, R. van Hezewijk y S. Terwee (Eds.), Recent Trends in Theoretical Psychology, Volume II (pp. 305-315). Nueva York: Springer. https://doi. org/10.1007/978-1-4613-9688-8_30

Vande Kemp, H. (2002). Making the history of psychology clinically and philosophically relevant. . History of Psychology, 5, 224-239. https://doi.org/10.1037/1093-4510.5.3.224

Vaughn-Blount, K., Rutherford, A., Baker, D. \& Johnson, D. (2009). History's Mysteries Demystified: Becoming a Psychologist-Historian. American Journal of Psychology, 122,, 117-129.

Vázquez-Ferrero, S. (2010). Presencia e Impacto del Psicoanálisis en Asignaturas Avanzadas de la Licenciatura en Psicología en la UNSL. Psiencia, 2, 55-59.

Vázquez-Ferrero, S. (2011). Autores predominantes en asignaturas cognitivas en la Universidad Nacional de San Luis: estudio bibliométrico. En Memorias del III Congreso Internacional de Investigación y Práctica Profesional en Psicología y Séptimo 
Encuentro de Investigadores en Psicología del Mercosur (pp. 89-92). Buenos Aires: Universidad de Buenos Aires.

Vázquez-Ferrero, S. (2016). Análisis de la bibliografía de programas vigentes en carreras de psicología de universidades nacionales argentinas (Tesis de doctorado inédita). San Luis: Universidad Nacional de San Luis.

Vázquez-Ferrero, S. \& Colombo, R. (2008). Undergraduate psychology programs in Argentina: bibliometrical analysis of texts listed in the syllabo of 14 advanced courses at the National University of San Luis. International Psychology Bulletin, 12, 16-18.

Vera Ferrándiz, J. A. (2008). Puente de plata para un provechoso encuentro entre la nueva historia y la psicología contemporánea. Pretextos para un diálogo necesario. Revista de Historia de la Psicología, 29, 7-29.

Vezzetti, H. (1998). Las psicologías del fin de siglo a la luz de su historia. Revista de Psicología General y Aplicada, 51, 105-114.

Vezzetti, H. (2007). Historia de la Psicología: Problemas, funciones y objetivos. Revista de Historia de la Psicología, 28, 147-166.

Vilanova, A. (1987). La carrera de Psicología en la Universidad Nacional de Mar del Plata. Actualidad Psicológica, 12, 3.

Vilanova, A. (1987/2003). La psicología como ciencia y como profesión. En A. Vilanova (Comp.), Discusión por la Psicología (pp. 9-27). Mar del Plata: Universidad Nacional de Mar del Plata.

Vilanova, A. (1990). Historia de la psicología clínica. Boletín Argentino de Psicología, 3, 7-19.

Vilanova, A. (1993). La formación de psicólogos en Iberoamérica. Acta Psiquiátrica y Psicológica de América Latina, 39, 193-205.

Vilanova, A. (1995a). El Dilema Olvidado de la Psicología Latinoamericana. Cuadernos Argentinos de Historia de la Psicología, 1, 83-99.

Vilanova, A. (1995b). Pautas para un Perfil de Psicólogo Iberoamericano. En G. Vidal, R. Alarcón y F. Stepke (Eds.), Enciclopedia Iberoamericana de Psiquiatría (pp. 666-673). Buenos Aires: Panamericana. 
Vilanova, A. (1996). Enseñanza de la Psicología: Historia y problemas fundamentales. Cuadernos Argentinos de Historia de la Psicología, 2, 199-210.

Vilanova, A. (1997a). La Historia de la Psicología y su sentido curricular. Clepios, 3, 18-23.

Vilanova, A. (1997b). Las deudas de la psicología del cono sur. Acta Psiquiátrica y Psicológica de América Latina, 43, 103-111.

Vilanova, A. (2000). Diez Problemas en la Historia de la Psicología como Curso de Grado. En C. J. Ríos, R. Ruiz, J. C. Stagnaro y P. Weissmann (Comps.), Psiquiatría, Psicología y Psicoanálisis: Historia y Memoria (pp. 143-154). Buenos Aires: Polemos.

Vilanova, A. \& Di Doménico, C. (1999). La psicología en el Cono Sur. Datos para una historia. Mar del Plata: Martín.

Visca, J. \& Moya, L. (2013). Analisis bibliométrico de la bibliografía obligatoria de las cátedras de Historia de la Psicología de Universidades Públicas Nacionales. Actas del XIV Encuentro Argentino de Historia de la Psiquiatría, la Psicología y el Psicoanálisis, 14, 343-348.

Vorano, A., Tosi, L. \& Tosi, J. (octubre, 2016). Impacto del escolasticismo en la formación universitaria en psicología. Trabajo presentado en el II Congreso Latinoamericano para el Avance de la Ciencia Psicológica: Buenos Aires, Argentina.

Ware, M. \& Benjamin, L. (1991). The APA Centennial and Teaching History of Psychology. Teaching of Psychology, 18, 136-144. https://dx.doi.org/10.1207/s15328023top1803_2

Weidman, N. (2016). Overcoming our mutual isolation: How historians and psychologists can work together. History of Psychology, 19, 248-253. https://dx.doi.org/10.1037/hop0000042.

Woodward, W. (1980). Toward a Critical Historiography of Psychology. En J. Brozek y L. Pongratz (Eds.), Historiography of Modern Psychology (pp. 29-70). Toronto: Hogrefe.

Woodward, W. (1994). Beyond the White Male Canon: Teaching Postcolonial History of Psychology. Psychologie und Geschichte, 6, 200-2011. 
Woody, D. (2011). Engaging Students in History and Systems of Psychology Courses. En R. Miller, E. Balcetis, S. Burns, D. Daniel, B. Saville y D. Woody (Eds.), Promoting Student Engagement. Volume 2: Activities, Exercises and Demonstrations for Psychology (pp. 129-134). Recuperado de http://teachpsych.org/ebooks/ pse2011/index.php.

Young, R. (1966). Scholarship and the History of the Behavioural Sciences. History of Science, 5, 1-51. https://doi. org/10.1177/007327536600500101

Zehr, D. (2000a). Teaching Psychology's History Throught a Comparative Analysis of Introductory Psychology Texts. Teaching of Psychology, 27, 31-33. https://doi.org/10.1207/ S15328023TOP2701_6

Zehr, D. (2000b). Portrayals of Wundt and Titchener in Introductory Psychology Texts: A Content Analysis. Teaching of Psychology, 27(2), 122-126.

Zehr, D. (2004). Two Active Learning Excercises from a History of Psychology Class. Teaching of Psychology, 31(1), 54-56.

Ziman, J. (1986). Introducción al estudio de las ciencias. Barcelona: Ariel.

Recibido: 14 de marzo, 2017 Revisado: 21 de setiembre, 2017 Aceptado: 19 de octubre, 2017 